AperTO - Archivio Istituzionale Open Access dell'Università di Torino

\title{
Interplay Between Mechanochemistry and Sonochemistry
}

\section{This is the author's manuscript}

Original Citation:

Availability:

This version is available http://hdl.handle.net/2318/1573165

since 2020-01-25T07:37:01Z

Publisher:

Boulatov, $\mathrm{R}$

Published version:

DOI:10.1007/128_2014_623

Terms of use:

Open Access

Anyone can freely access the full text of works made available as "Open Access". Works made available under a Creative Commons license can be used according to the terms and conditions of said license. Use of all other works requires consent of the right holder (author or publisher) if not exempted from copyright protection by the applicable law. 
This is the author's final version of the contribution published as:

Cintas, P; Cravotto, G; Barge, A ; Martina, K. Interplay Between

Mechanochemistry and Sonochemistry. Boulatov, R. 2015. pp: 239-284.

in

\section{POLYMER MECHANOCHEMISTRY}

When citing, please refer to the published version.

Link to this full text:

http://hdl.handle.net/2318/1573165 


\section{THE INTERPLAY BETWEEN MECHANOCHEMISTRY SONOCHEMISTRY}

Pedro Cintas ${ }^{1 *}$, Giancarlo Cravotto $^{2 *}$, Alessandro Barge $^{2}$, Katia Martina $^{2}$

${ }^{1}$ Departamento de Química Orgánica e Inorgánica, Universidad de Extremadura, Avenida de Elvas s/n, E-06006 Badajoz, Spain.

2 Dipartimento di Scienza e Tecnologia del Farmaco and NIS - Centre for Nanostructured interfaces and surfaces, University of Turin, Via P. Giuria 9, 10125 Turin, Italy.

\section{Table of Contents}

Abstract

1. Introduction: Sound and Energy

2. Cavitation and Secondary Mechanical Effects

3. Assembly and Scission in Molecular and Supramolecular Arrangements

3.1. Organogel and Hydrogel Formation

3.2. Exfoliation of Layered Materials

3.3. Modification of Polymeric Structures

4. Mechanical Action on Biostructures

5. Ultrasonically-driven Motion

6. Cleaning, Erosion, and Streaming: New Developments

7. Concluding Remarks: Open Questions

Acknowledgments

References 


\begin{abstract}
Ultrasonic irradiation based mechanochemical strategies have recently been the subject of intensive investigation because of the advantages they offer. These include simplicity, energy savings and wide applicability. Traditional areas of sonoprocessing such as cleaning, efficient mixing and solid activation have been extended to both macromolecular and micro/nanostructures, some of which are biologically significant, ultrasound-responsive actuators and crystal design, among others. Unlike conventional mechanochemical protocols, which require little solvent usage if any at all, mechanical (and chemical) effects promoted by ultrasound are observed in a liquid medium. Tensile forces, which share similarities with solid mechanochemistry, are generated by virtue of nonlinear effects, notably cavitation, when high-amplitude waves propagate in a fluid. This work aims to provide insight into some recent developments in the multifaceted field of sono-mechanochemistry using various examples that illustrate the role of ultrasonic activation, which is capable of boosting hitherto sterile transformations and inventing new crafts in applied chemistry. After a preliminary discussion of acoustics, which hopes to provide a mechanistic background, we shall mainly focus on experimental developments, while we will often mention emerging science and occasionally delve into theoretical models and force simulations.
\end{abstract}

Key words: cavitation, mechanical effects, mechanotransduction, self-assembly, streaming, ultrasound-responsive systems

\title{
1. Introduction: Sound and Energy
}

Sound is essentially mechanical energy. This simple assumption, well known by physicists and acousticians in particular, is however overlooked (if not ignored) by other scientists [1,2]. A sound wave propagating in air or any other fluid is a compressional wave, which can be characterized by a series of properties like frequency, pitch and intensity. The latter is the average power transmission per unit area perpendicular to the direction of the wave. The unit is the bel (B), although a unit which is ten times smaller, the decibel $(\mathrm{dB})$ is commonly employed. Conversational speech has an intensity of $c a .60$ $\mathrm{dB}$ at a distance of about $1 \mathrm{~m}$ from the mouth, while traffic in a noisy street can supply ca. 75-80 dB. The propagation of sound may be regarded as a form of transmission of energy through matter. Thus work is done (and hence energy is expanded) when a fluid is disturbed at a point. The reappearance of the disturbance (compression) at another point corresponds to energy transfer via wave propagation (sound possesses longitudinal nature). Following on from the preceding statements, average energy transfer rate per unit time per unit area of the wave front is also the intensity. In practice it is expressed as the average flow of power per unit area, whose unit is the watt per square centimetre $\left(\mathrm{W} / \mathrm{cm}^{2}\right.$ ). The lowest amount of sound energy humans can hear is about $10^{-20} \mathrm{~J}$ (or in 
other words, a minimum audible intensity of $c a \cdot 10^{-16} \mathrm{~W} / \mathrm{cm}^{2}$ ). The upper limit of sound intensity that can be generated is set by atmospheric pressure and such a wave would have an intensity of approximately $190 \mathrm{~dB}$. The mathematical analysis shows that the intensity of a plane sound wave is given by:

$I=p^{2}{ }_{\text {emax }} / 2 \rho_{o} \mathrm{~V}$

in which $p_{\max }$ is the maximum excess pressure in the wave, $\rho_{o}$ is the average (equilibrium) density of the medium and $\mathrm{V}$ is the sound velocity. It is noteworthy that this expression is independent of frequency. For dry air at $20{ }^{\circ} \mathrm{C}$ and standard atmospheric pressure, the equilibrium density $\left(\rho_{o}\right)$ is only $0.001205 \mathrm{~g} / \mathrm{cm}^{3}$ and $\mathrm{V}=344 \mathrm{~m} / \mathrm{s}$. When such values are substituted into the above equation and then converted to $\mathrm{W} / \mathrm{cm}^{2}$, an intensity $(I)$ of about $10^{-9} \mathrm{~W} / \mathrm{cm}^{2}$ results, or $70 \mathrm{~dB}$ above the minimum audible intensity. Also, it should be noted from the above equation that the intensity for given excess pressure depends on the medium and in particular on the $\rho_{o} \mathrm{~V}$ product. The latter is the specific acoustic resistance of the medium for a plane wave. For water under standard conditions, $\rho_{o} \mathrm{~V}$ is ca. 3800 times that for air. It takes an excess pressure ca. 60 times that for air to generate a comparable intensity.

Nonlinear effects, however, limit the maximum pressure that can be obtained. Sound waves of large amplitudes will ultimately break into a shock with morphologies that deviate from a sinusoidal curve (vide infra).

The term sonochemistry indicates the use of sound waves to generate chemical and physical effects that can be harnessed in multiple applications (Figure 1). Although such effects can be obtained at a wide range of frequencies, the word "sonochemical" is invariably linked to ultrasound, i.e. sound we cannot hear (typically above $20 \mathrm{kHz}$ ). Natural phenomena are good sources of both ultrasonic (e.g. animal communication or navigation) and infrasonic waves (such as earthquakes and tidal motion). Ultrasonics is current of interest for lay people because of medical imaging, metal cleaning, industrial and dental drills and non-destructive material characterization. 


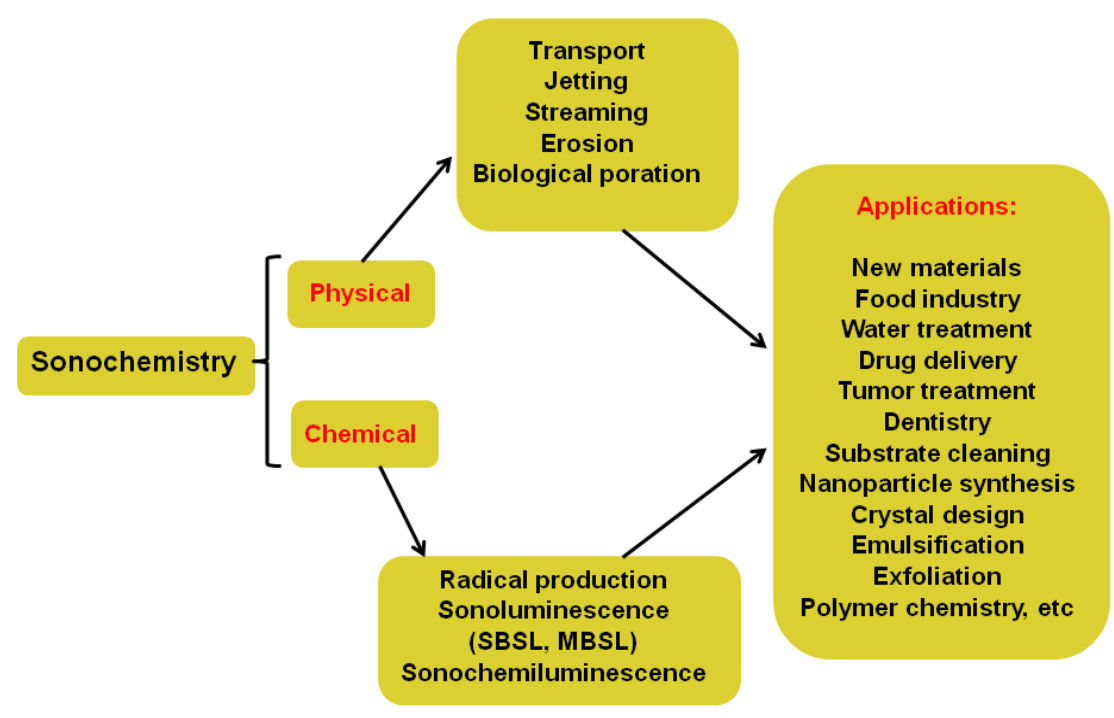

Fig. 1. Roadmap for sonochemical research.

Although cavitational effects were first identified in the late 1890s and were subsequently modelled by Lord Raleigh, ultrasound was no more than a scientific curiosity until around 1910 [1,3]. Technical interest grew during WWI after the successful development of piezoelectric transducers in early forms of sonar to detect submarines. The field of ultrasonics can be divided into two large domains; low-energy waves and high-energy waves. In the first case, the amplitude (i.e. the height of the wave from its highest point to its lowest point) is low enough so that the wave is not appreciably distorted in passing through the medium, which remains largely unchanged except for a slight rise in temperature (due to variation in intensity, directly proportional to wave amplitude). For high-energy waves, however, physical and chemical effects occur as the medium undergoes changes caused by the generation of significant mechanical stress and/or intense local heating. The interaction between high-energy sinusoidal waves and a medium causes distortion and leads to important nonlinear effects which fall into the admittedly difficult field of physical acoustics. Such effects include shock-wave formation, acoustic streaming, cavitation and acoustic levitation. Both mechanical and chemical effects of interest and benefit to the molecular (or supramolecular) world arise from cavitation (Sect. 2). This quasi-adiabatic phenomenon entails the rapid nucleation, growth and implosion of microbubbles in a liquid that releases an enormous amount of kinetic energy which actually drives a chemical reaction to completion. This is different to a direct interaction between ultrasound and matter [4]. A more detailed description follows; pressure in a liquid will fall (below zero) sharply as an ultrasonic wave of sufficient intensity (i.e. acoustic pressure) exceeds the normal average pressure in a liquid. The liquid then ruptures and generates small cavities that 
will become unstable and collapse quickly. In the end, the gas entrapped within the cavity will be highly compressed (hydrodynamic models point to several hundreds of atmospheres and temperatures as high as $5000 \mathrm{~K}$ ). Such high pressures are relieved by the accompanying radiation of shock waves. As already mentioned, shock waves are a nonlinear effect which arise from explosions (e.g. cavitational collapse) or which are emitted from an object moving faster than sound.

Bubble collapse generates a disturbance that starts out with a sinusoidal curve, given by a linear equation, but soon develops into a sawtooth or triangular curve when excess pressure is plotted versus distance in the direction of wave propagation (Sect. 2). This wave will show discontinuities in pressure, density and flow velocity, which all become large changes over very small liquid intervals (about $10^{-4} \mathrm{~cm}$ ). As a result, the discontinuities travel through the medium faster than the ambient acoustic velocity. Mechanical effects thus caused by shock waves, liquid jets and shear forces in the bulk medium and bubble vicinity will result in enhanced mass and energy transfer, particle size reduction, liquid emulsification and surface activation. Purely chemical effects, such as molecular sonolysis, which leads to discrete radicals or excited species, polymer rupture and changes in solvation and ligand-metal coordination, will mainly occur inside the bubbles (so long as reagents possess sufficient volatility) or at the bubble interface. Given the complex nature of acoustic cavitation, both mechanical and chemical effects are juxtaposed and may work cooperatively (Figure 2). Accordingly, a clear-cut dissection of mechanical effects is problematic, although they may be prevalent at suitable frequencies and intensities, as we shall see later.

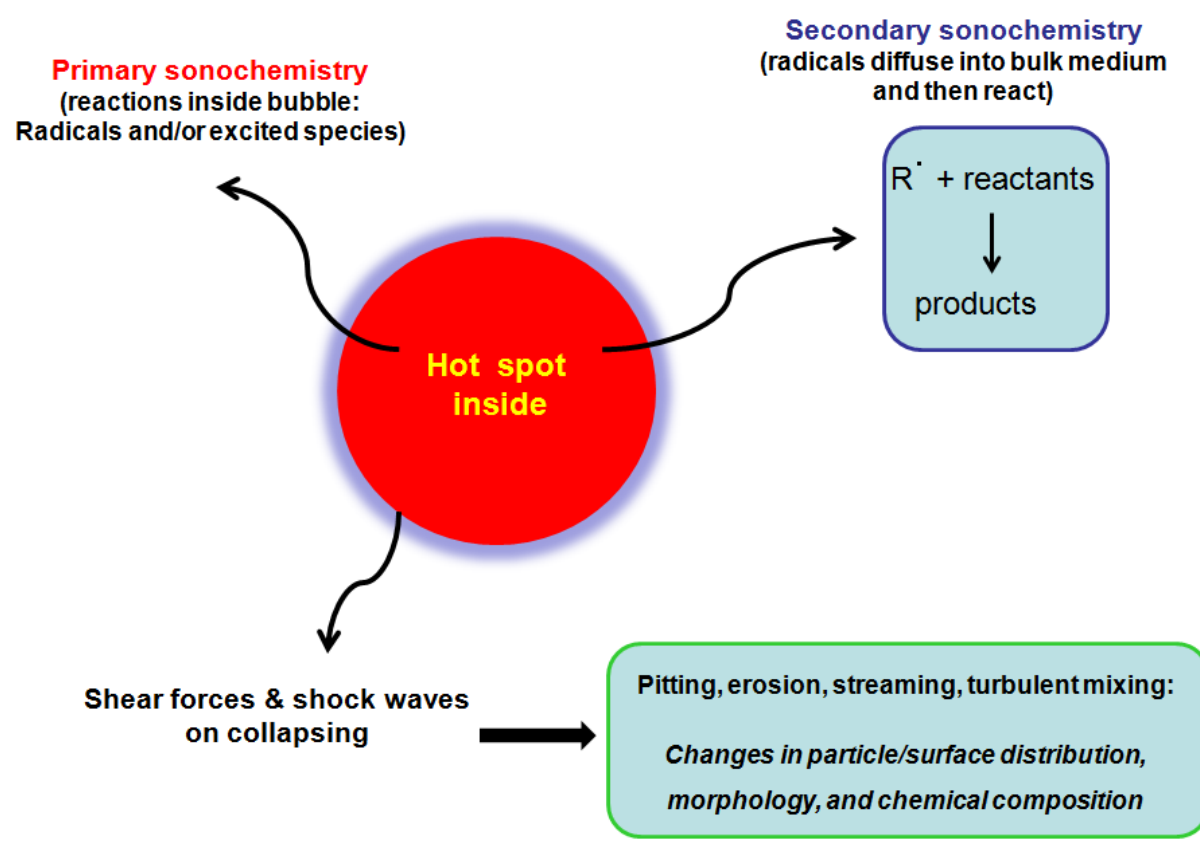

Fig. 2. Primary and secondary effects of acoustic cavitation. 
This chapter aims to describe the mechanical bias of cavitational effects and how they are related to conventional mechanochemistry and force-induced physical fields in general. This subject has been well documented over the last decade [5-9], and particular attention has been paid to scenarios such as mechanically responsive polymers [10-12], micro- and nano-structured materials [13-15], and sonocrystallization [16], which are all expected to have a major impact on the fabrication of novel and smart materials as well as the pharmaceutical industry. Although we shall briefly mention these past achievements, our goal is to focus on the aspects of sono-mechanochemistry that have been overlooked in recent thematic issues. We will emphasise soft matter, both synthetic and biological, as well as the less-well-known, yet hugely promising, applications of acoustic fields, like $\mathrm{CO}_{2}$ capture and acoustically-driven micro- and nano-motors. Mechanistic frameworks should be investigated in these cases, although, unfortunately, space limitations prevent in-depth analysis. Our readers are referred to recent and comprehensive works such as those by Boulatov and associates [17], and RibasArino/Marx [18]. They largely deal with attempts to understand chemical reactivity under mechanical loads in general and some models may certainly be extrapolated to ultrasound-induced forces. Much more futuristic areas, where a coupling of sound wave mechanical energy and nonlinear effects will certainly provide both a technological and societal impact, can be found in the creation of acoustic metamaterials and phononic crystals [19-22]. These issues however lie beyond the bounds of this article.

\section{Cavitation and Secondary Mechanical Effects}

As mentioned in the introductory remarks, ultrasound waves transport both kinetic energy (particles of the medium oscillate and displace from their equilibrium position in the direction of propagation) and potential energy (fluid compression) as fluids can support negative pressure for short times. When a sufficiently large negative pressure is applied to the liquid (here it is the pressure on rarefaction) so that the distance between the molecules exceeds the critical molecular distance to hold the liquid intact, the latter will inevitably break down and microbubbles will form. The characteristic linearity of sinusoidal acoustic waves is no longer valid at high amplitudes and nonlinear phenomena cause sudden changes in pressure and fluid properties, such as the generation of shock waves. The rate of pressure change is determined by sound attenuation in the medium, which also depends on frequency. In water, the shock rise time (i.e. the time interval for the wave to vary from $10 \%$ to $90 \%$ of its peak value) may be much shorter than the wave period whilst for other materials, such as biological tissues, the pressure variations will be less pronounced. Figure 3 shows numerical predictions of waveform distortion produced by nonlinear propagation, at different sound attenuations, in water and homogeneous tissues. The waveforms are generated in the focal region at a range of $52 \mathrm{~mm}$ from a rectangular 3.0-MHz [23]. The waveforms lack symmetry and tend to have a shorter compression phase followed by a longer rarefaction phase. The overall effect is a 
reduction in rarefaction pressure, with respect to linear propagation, leading to the generation of harmonics which may extend to many times the original frequency.

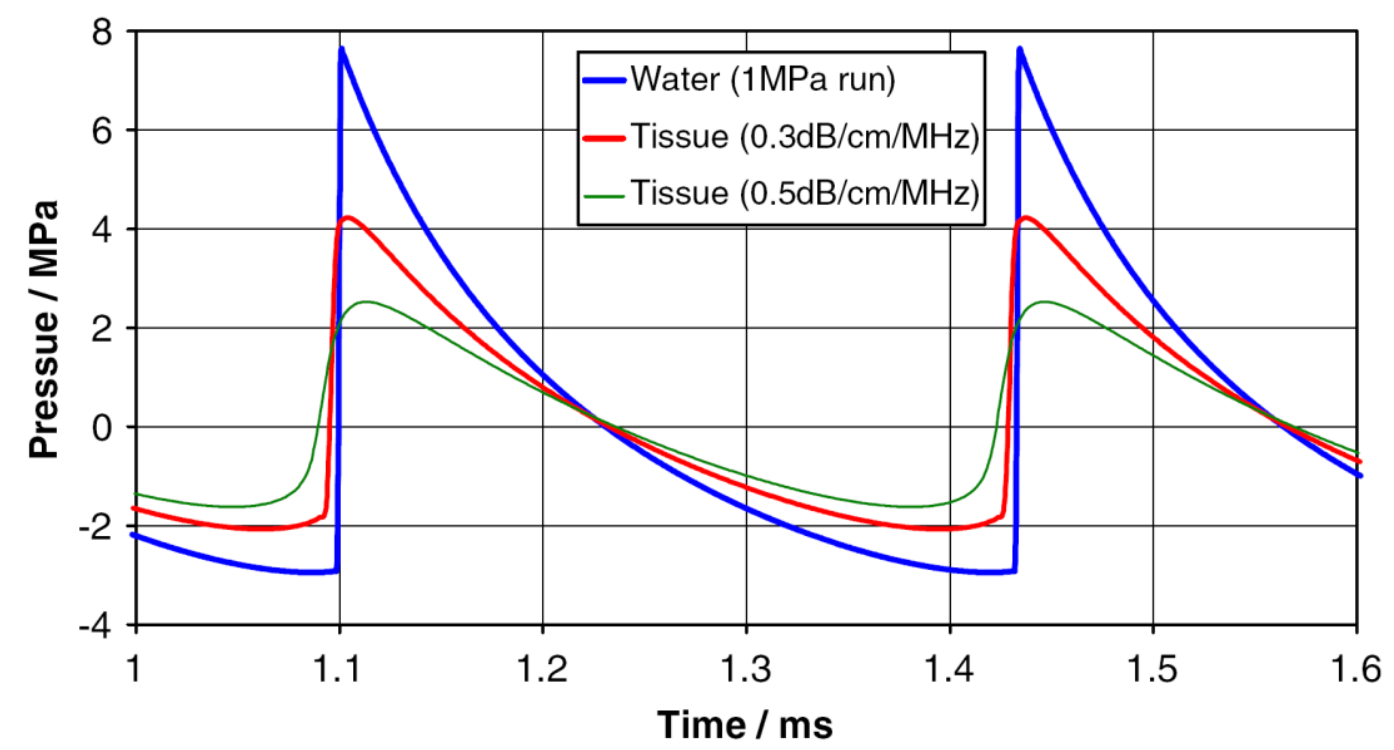

Fig. 3. Model predictions of shock waves from a transducer $(3.0 \mathrm{MHz})$ focused at 70 $\mathrm{mm}$, propagating in water and homogeneous tissues at different sound attenuation values. The average amplitude at the face of the transducer is $1.0 \mathrm{MPa}$. Reproduced with permission from ref. [23] Copyright 2006 Elsevier Ltd.

Enhanced sound attenuation can also lead to other secondary effects that include local heating and streaming. Both radiation pressure and streaming are prominent phenomena associated with the propagation of ultrasonic waves [23,24]. These subjects require a significant mathematical background, which may be tedious and/or mislead the readership of this article. It suffices to firstly introduce the concept of excess pressure and then recall previous statements. For a harmonic wave in a fluid, its displacement $(\xi)$ can be replaced by the excess pressure $\left(p_{\mathrm{e}}\right)$ generated by the wave, which is defined by the difference between the actual pressure $(p)$ at any point and time and the normal pressure $\left(p_{\mathrm{o}}\right)$ in the initially undisturbed fluid. Thus, $p_{\mathrm{e}}=p-p_{\mathrm{o}}$ and that excess pressure gives spatio-temporal variations like $\xi$ in the harmonic equation:

$\xi=\mathrm{A} \sin 2 \pi(f t-x / \lambda)$

where $\mathrm{A}$ is the amplitude, $f$ the frequency, $t$ the elapsed time, $x$ the distance from the origin and $\lambda$ the wavelength. For plane harmonic waves the excess pressure will vanish over time, although this premise only holds for low intensity sound. At higher intensity, excess pressure that does not become zero on average is also present, thereby making a net contribution to the static pressure prevailing in the fluid, which is known as the 
radiation pressure. In a simplified form, radiation pressure can be roughly expressed as the ratio of the intensity of the sound $(I)$ to its velocity $(c)$, i.e.:

$P_{\mathrm{rad}} \cong I / c$

Radiation pressure is a steady constant pressure for continuous wave signals. For acoustic pulses however, that pressure varies periodically at the pulsing frequency. Radiation pressure thus provides a mechanism for producing force at frequencies other than the normal ultrasonic frequencies and potentially in the audible zone. For small particles and non-absorbing interfaces, radiation pressure will have a direction and amplitude which depend on the elastic properties of the material in question. This extra force may also result in particle movement.

Likewise, acoustic streaming stems from high-amplitude waves travelling through a medium. In linear behaviour, particles are expected to vibrate about their equilibrium position; in other words, with no net flow. In stark contrast to this and as a result of ultrasonic waves' attenuation with distance, there will be a pressure gradient in the fluid that gives rise to a net flow. The magnitude and pattern of the flow depends on the wave properties as well as the type of fluid and reaction vessel. The flow usually moves axially away from the transducer with recirculation vortices that bring fluid back to the transducer face. Visualization of streaming in liquids can easily be accomplished using thymol blue indicator [23-25]. The maximum axial streaming velocity $\left(v_{\max }\right)$ for a focused beam of intensity $I$ and radius $r$, can be approximately estimated as:

$\mathrm{v}_{\max } \cong \alpha I r^{2} G / c_{\mathrm{o}} \mu$

where $\alpha$ is the amplitude attenuation coefficient, $\mu$ is the shear viscosity of the fluid and $G$ a constant that depends on beam characteristics and vessel geometry. It is convenient to recall that sound attenuation involves the loss of wave amplitude due to different mechanisms such as absorption or scattering. Attenuation is measured in dB per unit length and also depends on the frequency of the incident beam. Accordingly, the attenuation coefficient $(\alpha)$ is usually given in $\mathrm{dB} /(\mathrm{kHz}$ or $\mathrm{MHz}) \cdot \mathrm{cm}$.

Globally considered, fluid streaming developed around a solid particle or surface greatly increases both mass and heat transfer. The sonoprocessing of fluidized beds is an important application with environmental connotations (Sect. 6), as the entrainment of fine particles in the sound wave improves fluidization in dense beds and alternatively induces particle agglomeration in diluted beds [26].

The above-mentioned mechanical effects can be significantly enhanced if cavitation takes place in the ultrasonic field. A threshold intensity, which in turn depends upon frequency, must be reached for cavitation to occur, although the process is largely 
influenced by external parameters, such as solvent properties, temperature or dissolved gases. Cavitation in liquids decreases as one moves to higher frequencies (e.g. the $\mathrm{MHz}$ region). This can easily be explained as the rarefaction (as well as compression) phase shortens. With shorter cycles, the finite time required for molecules to be pulled apart, thus generating a cavity, becomes too short. Accordingly, higher amplitudes (power) will be required to make liquids cavitate at high frequencies. The fact that most sonochemical applications are run between 20 and $100 \mathrm{kHz}$ is not a coincidence [4,27].

Sonochemists recognize two types of cavitation; stable and transient. The former means that bubbles oscillate around their equilibrium position, possibly over prolonged compression/rarefaction cycles. Stable cavitation simply emerges because the acoustic pressure serves as a force that drives the bubble and varies its radius. Experimental observations show that a pulsating bubble under stable cavitation grows progressively. This effect is associated with another phenomenon; rectified diffusion. During the compression phase, the gas inside the bubble is at a pressure higher than the equilibrium value and diffuses from the bubble. Conversely, the pressure within the cavity decreases during rarefaction, so that some gas diffuses into the bubble. Because of the larger area present during expansion than under compression, there is a net increase in gas content in the bubble over a cycle. Obviously, a bubble cannot grow indefinitely. During expansion, bubbles reach a critical size and then collapse violently because of the inertial forces of the spherically converging liquid [23,24]. This transient (or inertial) cavitation triggers well-established mechanical (shocks) and potential chemical effects (highly excited species formation or light emission = sonoluminescence) . The disturbance caused by the presence of pulsating bubbles will also generate a small fluid flow, i.e. microstreaming. Since this flow varies with distance from the bubble, high shear stress near the bubble also occurs and this mechanical action alone is sufficient to cause cell and soft tissue deformations or damage.

This discussion of cavitation certainly overlooks specific details, but it highlights how the nonlinear propagation of ultrasound causes inhomogeneities and physical effects that may have consequences for molecules present in the medium. It is also worth mentioning that suitable cavitation nuclei may also be created at the expense of existing bubbles in a liquid or formed in crevices in suspended particles where very small amounts of gas can be trapped. An illustrative picture of this cascade process which involves several mechanisms that account for the mechanical action of ultrasonic waves is supplied in Figure 4 [28]. 


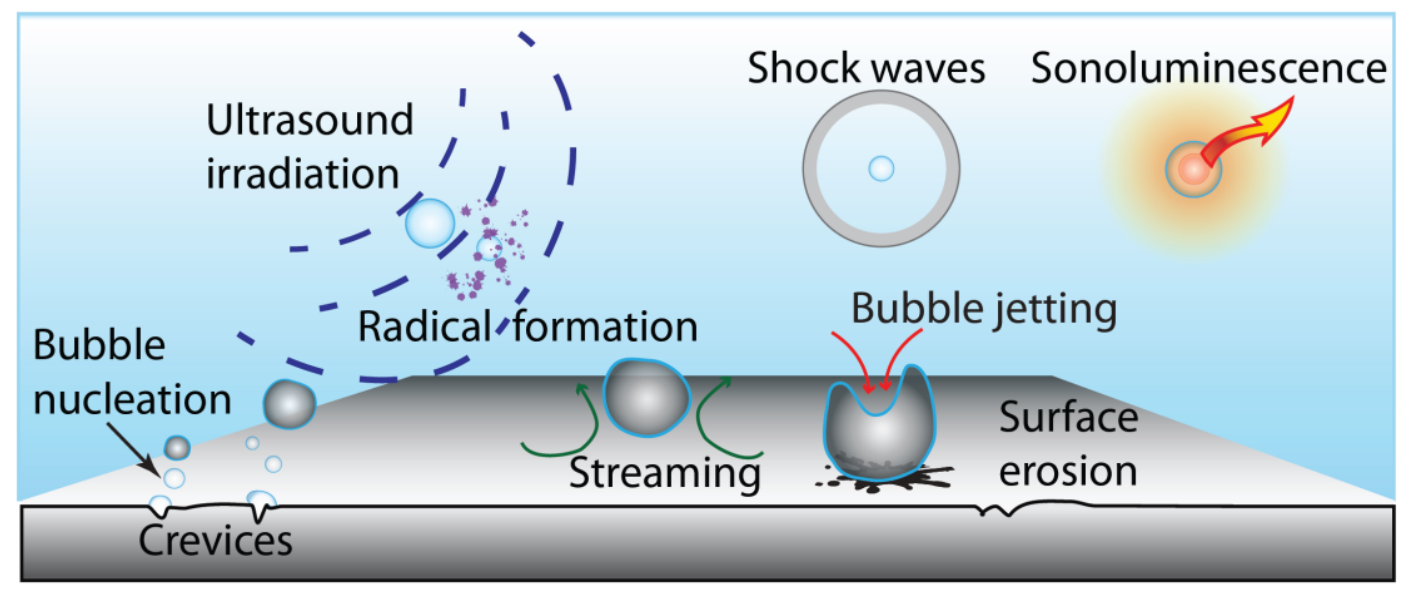

Fig. 4. Schematic representation of nonlinear effects produced by bubble collapse. Reproduced with permission from ref. [28]. Copyright 2012 David Fernández Rivas, Enschede, The Netherlands.

It is hoped that enhanced mechanical action will take place in association with high intensities and frequencies. However, harmonic generation and sound attenuation provide secondary mechanisms capable of inducing effects within a wide range of frequencies. An assessment of mechanical effects as a function of nominal frequency is not immediately obvious. Mason and associates have monitored, using SEM (scanning electron microscopy) and XPS (x-ray photoelectron spectroscopy), the abrasive action of sound waves on a glass-filled polystyrene blend by measuring weight loss at different frequencies $(20,40,582,863$, and $1142 \mathrm{kHz})$ [29]. At low frequencies $(20-\mathrm{kHz}$ probe or 40-kHz bath), the high weight loss correlated well with significant surface changes which were purely caused by mechanical effects, such as micro-jetting. Less pronounced changes occurred at higher frequencies, although surface oxidation was ascribed to enhanced radical specie production. These conclusions, attained in aqueous solutions, agree with previous observations on dextran degradation. Here, mechanical action was important at $35 \mathrm{kHz}$, whereas extensive degradation took place at $500 \mathrm{kHz}$ and higher frequencies due to radical reactions [30]. A recent study by Tran and coworkers furnished similar conclusions on the inverse dependence of mechanical and chemical effects on ultrasonic frequencies. Since power intensity (usually measured by calorimetry) decreases as frequency increases, these authors insisted on the use of constant power intensity. By measuring the degradation rates and viscosity ratios of poly(ethylene oxide) (in water and benzene) and polystyrene (in benzene only) under ultrasonic irradiation (from $20 \mathrm{kHz}$ to $1 \mathrm{MHz}$ ), this group found a decrease in mechanical effects above 100 $\mathrm{kHz}[31]$.

\section{Assembly and Scission in Molecular and Supramolecular Arrangements}


That ultrasonic waves promote both molecular association and the extensive disruption of structural arrangements may, at first sight, appear somewhat counterintuitive. Once again, such effects depend on the characteristics of the ultrasonic field. Numerical methods and simulations have been introduced to predict the active zones while bearing in mind that, by definition, a cavitating field is a heterogeneous system (liquid and bubbles) [32-34]. In general, reproducible results are attained as long as external factors and reactor shape are optimized, although some practitioners often ignore the critical issue of power control.

Since acoustic radiation lacks quantum character, mechanical oscillations can be harnessed at various frequency ranges, especially via large molecular structures which respond by triggering chemical and biochemical events. Surface acoustic waves (SAWs), for instance, which operate in the $\mathrm{MHz}$ zone have been used for the actuation of fluids, polymer patterning and the directional manipulation of particles on surfaces [35-37]. Atomistic explanations remain largely obscure in view of the frequency mismatch between SAWs and the vibrational states of the adsorbed species. The acoustic enhancement of surface diffusion has recently been suggested [38], which might also account for well-known cases of surface catalysis [39,40] and molecular desorption $[41,42]$. The experimental observation of ultrasonically-induced birefringence is also relevant $[43,44]$. On the other hand, low-frequency mechanical vibrations have proven to be useful in lattice crystallization as they favour higher ordering and packing $[45,46]$. The process depends on vibrational amplitude and frequency and is also related to the appearance of convective flow. Flow configuration and velocity can be altered by changing the acoustic parameters. The particle aggregation mechanism is herein described in terms of stochastic resonance, which contrasts with the usual approach of maintaining equilibrium conditions during crystallization [47]. Similar analogies can be encountered, within the broad context of mechanochemistry, in granules climbing along a vibrating tube, where the force between the tube and the particles varies periodically thus causing a directional effect [48].

\subsection{Organogel and Hydrogel Formation}

If there is a paradigmatic case of ultrasound-assisted formation and the structural manipulation of soft matter, it would most likely be gelation. The process is hardly new and can historically be ascribed to early observations of pattern formation in liquids and solids induced by mechanical vibrations, although the field has experienced a further renaissance over the last two decades, thus launching molecular level investigations [49]. Low-molecular weight (LMW) molecules (usually with molar masses below 3000 and not involving polymeric chains) are capable of immobilizing significant amounts of 
organic solvents or water, thereby serving as organogels or hydrogels, respectively. These substances self-assemble in a given solvent, via non-covalent interactions such as hydrogen bonding (which is probably the most prevalent intermolecular interaction), van der Waals interactions and $\pi-\pi$ stacking, which all cooperatively drive the onedimensional growth of LMW molecules, leading to fibres and strands. These further assemble into three-dimensional networks that encapsulate the solvent and impede flow. This kind of supramolecular soft material which responds to sonication and stress may find numerous applications in biomedical research and the preparation of self-healing substances or mechanical switches [50,51].

It is fair to recognize that the 2005 paper by Naota and Koori triggered subsequent developments in this discipline. The paper reported rapid gelation upon the sonication of solutions containing a dinuclear Pd-salicylidene complex, which is stabilized by intramolecular $\pi$-stacking interactions [52]. When solutions of complex anti-1 (Figure 5) were irradiated with ultrasound $\left(40 \mathrm{kHz}, 0.45 \mathrm{~W} / \mathrm{cm}^{2}\right)$ at room temperature, gelation took place within a few seconds. The resulting gels were thermoreversible and clear solutions were obtained via heating to temperatures above $T_{\text {gel }}$. Derivatives with longer hydrocarbon spacers $(n=6-8)$ failed to gelate, although precipitation was sometimes observed. The process also exhibited marked stereoselectivity as the syn isomer of $\mathbf{1}$ did not give gels under the same conditions. These complexes also show planar chirality and this feature was instrumental in elucidating the putative role of ultrasound. When enantiopure anti-1 was subjected to prolonged sonication, it remained unaffected in solution. By stark contrast, scalemic mixtures of anti-1 (42\% ee) gave gelation, although the resulting gel showed no optical activity. In other words, gelation proceeds with racemisation. This points to a process involving the heterochiral association of $(R)$ - and $(S)$-configured monomers that alters the cofacial bent structure of the parent isomer. Without sonication, the conformation is stable enough and avoids aggregation, but irradiation leads to interpenetrative and consecutive planar monomers stacking (Fig. 5).
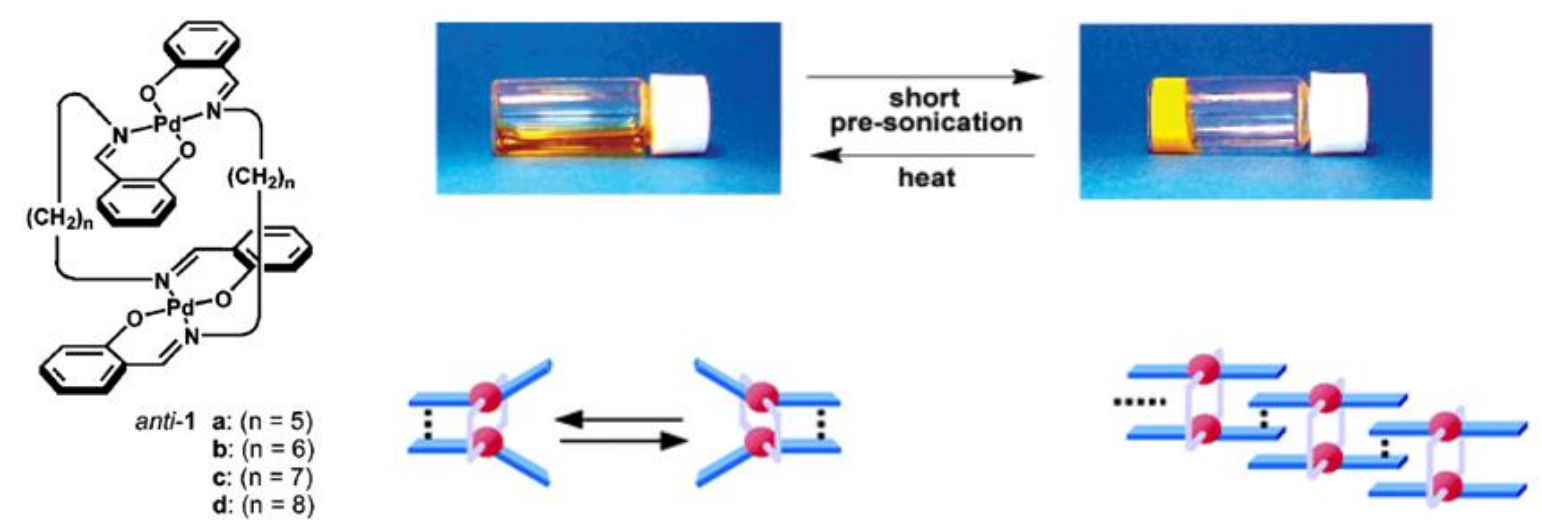

Fig. 5. Reversible sol-gel transformation of Pd-salicylidene complexes. Sonication favours gelation and induces a conformational change, from bent to interlocked 
structures. Reproduced with permission from ref. [52]. Copyright 2005 American Chemical Society.

The advantages and versatility of this type of gelation, which allows the synthesis of complex structures to be carried out, is illustrated by the incorporation of quantum dots (QDs) into hydrogels [53]. Thus, hexane suspensions of a dipeptide derivative and $\mathrm{CdSe} / \mathrm{ZnS}$ covered with trioctylphosphine oxide, as a surface ligand, undergo gelation in minutes under sonication. The resulting gels are luminescent under UV irradiation and can revert into the sol state upon heating and slow cooling (Figure 6). The procedure can be used for vapour sensing in the gel state, as shown for 2-mercaptoethanol (Fig. 6, bottom). The gel was prepared using $\mathrm{QD}_{598}$ and excess dipeptide in decane after 2 min of sonication. The QD-doped gel was then exposed to 2-mercaptoethanol vapours, as a function of time, under UV irradiation. The increase in photoluminescence can easily be detected over time as the gel becomes progressively more fluorescent as the QD reagent diffuses inside the organogel from the bottom to the top of the vial.
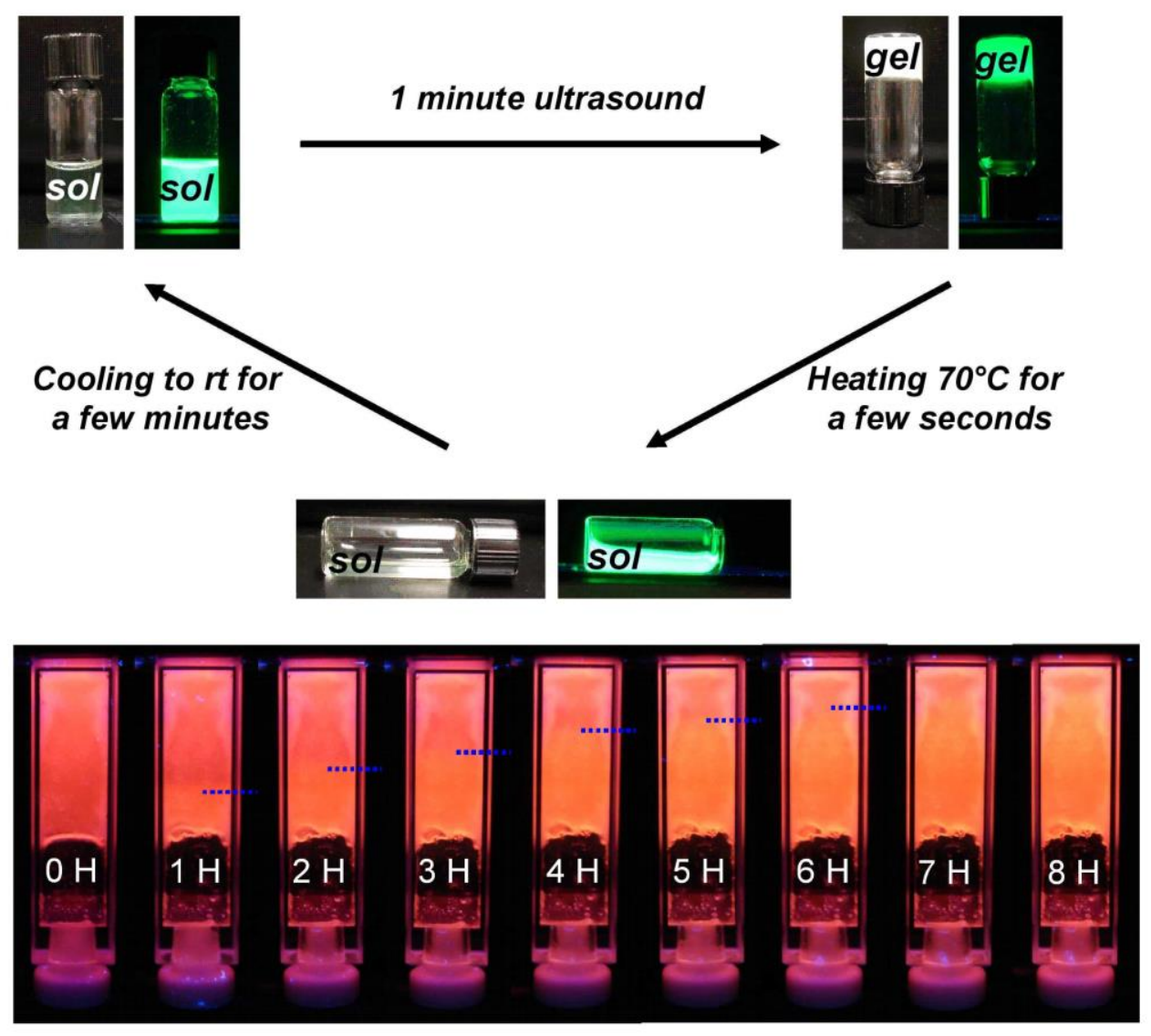

Fig. 6. Top: Hexane solutions containing a dipeptide plus QD524 undergo gelation after 1min of irradiation, followed by heating and slow cooling. The procedure can be repeated 
as the dipeptide precipitates at the bottom of the vial. Bottom: Use of ultrasoundgenerated gels containing QD 598 for vapour sensing (2-mercaptoethanol). Reproduced with permission from ref. [53]. Copyright 2008 Wiley-VCH Verlag GmbH \& Co. KGaA.

That sonication and shearing stress stimulates gelation, rather than structural disruption, is in principle challenging from a mechanistic point of view. Analysis is complicated because most experimental protocols do not always indicate intensity measurements. Nor do they report other parameters that affect the acoustic field. However, unlike gels controlled by chemical stimuli, mechanical force and moderate sonication do not generally modify (or destroy) the structure of a molecule in the gel network. The role of sonication appears to be twofold [50]. Firstly, ultrasound can transform intramolecular interactions into favourable intermolecular interactions that lead to aggregation. Secondly, sonication may induce conformational changes on the resulting organogel, i.e. after formation of the supramolecular network assembled by non-covalent interactions. This aspect is noticeable in the case of two-component organogels as irradiation often results in strengthening infinite hydrogen bonding and other intermolecular interactions that assist gel formation. The latter is well exemplified by melamine-uric acid hydrogels. Neither of the parent molecules is appreciably soluble in water, but gels formed at or above $0.8 \mathrm{wt} \%$ concentrations after sonication and shaking over short times (ca. $5 \mathrm{~min}$ ) [54]. Likewise, sonication triggers morphological changes such as the formation of fibres from a polypeptide organogelator that exists as vesicles in solution. This morphological switch is reversible and repeatable and the fibre-vesicle transition occurs on incubating the gel at $60{ }^{\circ} \mathrm{C}$ [55]. Similar morphological variations have been reported for a multifunctional adamantane-based gelator, for which 5-min irradiation affords partial gelation (in the form of vesicles), while prolonged sonication gives rise to a 3D-network. Again, the process is reversible over a wide concentration range via sonication and heating-cooling cycles. Gelation can also be fine-tuned by further host-guest interactions in the presence of $\beta$-cyclodextrin under sonication, with structures evolving from nanoribbons to ordered nanothreads [56].

A fibrillar structure appears to be the dominant and terminal motif in supramolecular gels generated by shear forces or sonication in liquids. This holds additional significance in biomimetic studies. For instance, silica nanofibers $(\sim 30 \mathrm{~nm}$ average diameter and $\sim 100 \mu \mathrm{m}$ length) have been recently obtained via the sonication of an emulsion containing tetraethyl orthosilicate as the silica precursor [57]. The protocol is relatively inexpensive, conducted in a conventional ultrasonic bath and overcomes difficulties associated with other methods like electrospinning or heating. SEM images show that nanofibres grow on the tips of silica rods, with both co-existing in the same solution (Figure 7). Only silica rods would be obtained in the absence of sonication, thus showing that irradiation does effectively promote fibrillation. The authors largely attributed this improved preparation to fluid motion created by ultrasound (i.e. microstreaming). Apparently, silica rods act as a micromotor and the torque produced by 
sound waves results in rod rotational motion, thereby spinning off nanofibres at the rod tips.
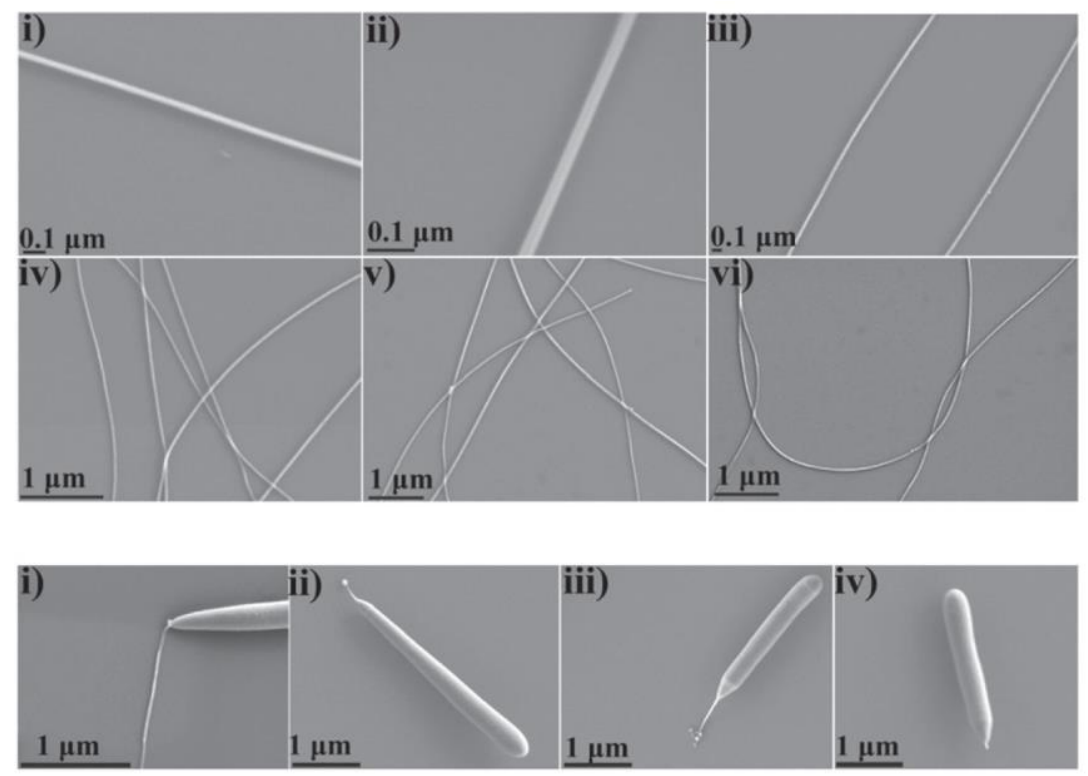

Fig. 7. Scanning electron microscopy (SEM) images of silica nanofibres (i-iii zoom in and iv-vi zoom out images) and a visualization of nanofibre growth on rod tips. Reproduced with permission from ref. [57]. Copyright 2014 The Royal Society of Chemistry.

The influence of ultrasonic force on aggregation morphologies can be obtained from crystallization experiments, especially en route to fluorescent materials with enhanced emission [58,59]. The striking conclusion reached is that luminescent properties are tuneable and can be controlled by molecular packing, which is greatly affected by the ultrasonic treatment and sensitive to its power. At low-energy ultrasonic power, a polar molecule, namely 4-[bis(4-aminophenyl)amino]- $N, N$-diphenylbenzamide (ITPADA), containing imide and amino groups, with spherical structure undergoes selfassembly via intermolecular hydrogen bonding that is stronger along $x$ and $y$ axes than along the $z$ axis. When the ultrasonic power increases, the layer-by-layer structure would be peeled off (see next subsection). As a result, the initially generated four-side prismatic structure becomes thinner and thinner, as power increases, and converts into a rhombic nanosheet array (Figure 8). Theoretical conclusions corroborate this finding as ITPADA molecules in single crystals lack coplanarity, due to intermolecular interactions, and their band gaps were accordingly higher $(>4 \mathrm{eV})$ than those of free molecules $(\sim 3.7 \mathrm{eV})$, thus accounting for blue-shifted fluorescence wavelengths [59]. 

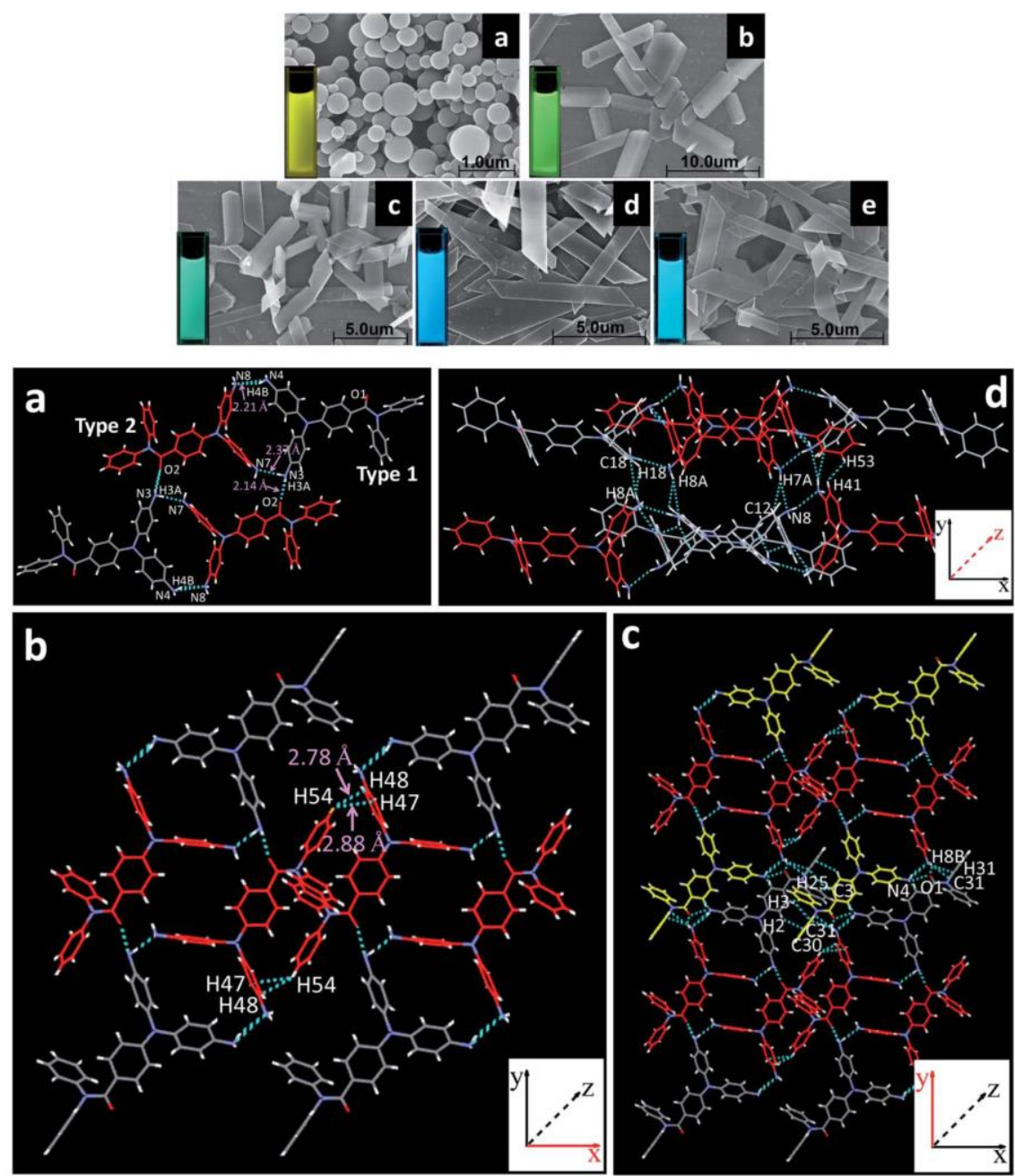

Fig. 8. Top: Influence of US power $(40 \mathrm{kHz})$ on the morphology and fluorescence color of ITPADA suspensions in THF- $\mathrm{H}_{2} \mathrm{O}$ mixtures (90\% water content): (a) non-ultrasonic; (b) $80 \mathrm{~W}$; (c) $120 \mathrm{~W}$; (d) $160 \mathrm{~W}$; (e) $200 \mathrm{~W}$. Bottom: Crystal packing of ITPADA single crystals: (a) basic unit of single crystals; (b) interactions along the $x$-axis; (c) interactions along the $y$-axis; (d) interactions along the $z$-axis. Reproduced with permission from ref. [59]. Copyright 2014 The Royal Society of Chemistry.

\subsection{Exfoliation of Layered Materials}

With the advent and fast development of graphene as the flagship material for futuristic technology, including interfaces with biology [60], the search for economic and large-scale syntheses of few-layer (ideally monolayer) graphene and other carbonaceous structures has become a front-line objective. Exfoliation is clearly a compulsory step and this goal can be achieved by numerous physical and chemical methods that can be extended to layered structures other than graphite, such as $\mathrm{BN}, \mathrm{MoS}_{2}, \mathrm{MoO}_{3}$ and clays 
[61]. Ultrasonic exfoliation is routinely performed in numerous solvents and then applied to a variety of chemical modifications [62], although the characterization of the ultrasonic field (power in particular) is not explicitly mentioned in most cases. As expected, bubble collapse generates mechanical force which is able to disrupt the weak intermolecular interactions present in layered materials (e.g. stacked graphene monolayers in the case of graphite).The liquid-phase exfoliation of graphene has recently been accomplished by means of a high-shear mixer, as long as the local shear rate exceeds $10^{4} \mathrm{~s}^{-1}$ [63]. In this context, parallelisms with ultrasound-induced shear forces should be scrutinized in terms of acoustic power [64], as exfoliation and dispersion depend on inertial cavitation and not on the stable stages [65]. Short ultrasound times at lower power generally produce highquality graphene layers with defects that are mainly located at layer edges. Prolonged irradiation is however detrimental and causes bulk defects, as shown by Raman spectroscopy in a recent study conducted in $N$-methyl-2-pyrrolidone (NMP), a typical solvent for graphene exfoliation. Such defects are essentially topological, while vacancies, atomic substitutions and $\mathrm{sp}^{3}$-like defects should be ruled out [66].

Together with sonication, microwave (MW) irradiation enables the exfoliation of graphene precursors leading to graphene itself [67] and other forms of porous carbon [68]. Some effort has also been expended on the combined use of MW and ultrasound, either combined or separately, for the purification and surface functionalization of layered materials such as carbon nanotubes. In a study aimed at evaluating both mechanochemical and frequency effects in the oxidation of multi-walled carbon nanotubes (MWCNTs), some conclusions were inferred from TEM (transmission electron microscopy) and Raman spectroscopy data [69]: a) MW irradiation causes rupture, because of fast heating and metal content, and the surface becomes highly oxidized (with $\mathrm{OH}$ and $\mathrm{COOH}$ groups); b) the combined use of MW and US leads to surface oxidation along with structural defects, although this protocol efficiently removes the amorphous external layer; c) sonication $(20 \mathrm{kHz})$ accelerates surface oxidation and damage, although partial exfoliation was observed (graphene sheets are noticeable), and d) at high frequency $(300 \mathrm{kHz})$, ultrasound promotes oxidation, but the tubular arrangement of CNTs is preserved (Figure 9).

Emerging hyphenated techniques, such as the use of MW and ultrasound beams, offer substantial rewards. The sequential approach mentioned above provides inherent advantages as sonication enables rapid exfoliation, while the chemical derivatization of graphene sheets is largely improved under MW irradiation. This strategy can be exemplified in the synthesis of graphite halides (Figure 10) [70]. The first step (halogenation reaction), involving the intercalation of the halogen between the graphite layers, is accelerated by microwaves so that the modified material can then be easily exfoliated into monolayer graphene in organic solvents under mild sonication. Alternatively, stable suspensions of few-layer graphene have been obtained via the 
sonication of graphite flakes in benzylamine which were then subjected to cyclopropanation under MW irradiation [71].

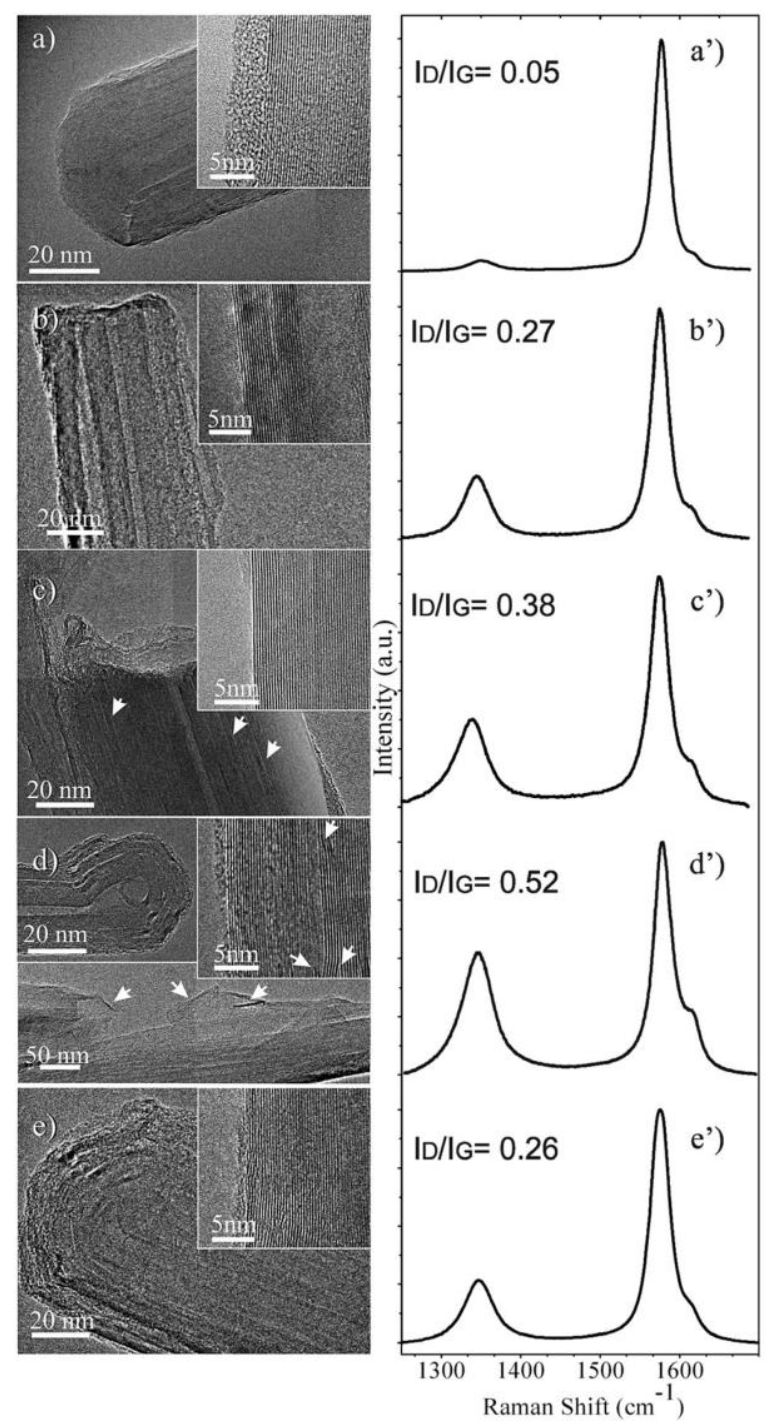

Fig. 9. TEM images and D/G Raman bands for (a) pristine MWCNT, (b) after MW irradiation $(2.45 \mathrm{GHz})$, (c) after sequential MW-US (20 kHz) irradiation, (d) sonication at $20 \mathrm{kHz}$, and (e) sonication at $300 \mathrm{kHz}$. Reproduced with permission from ref. [69]. Copyright 2011 The Royal Society of Chemistry. 


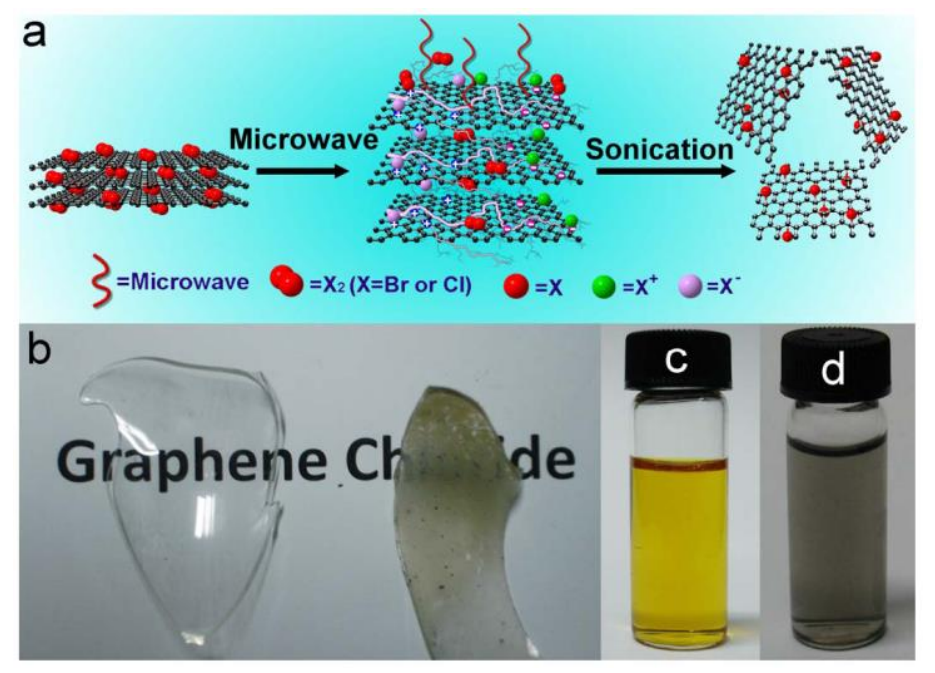

Fig. 10. (a) Scheme showing sequential MW-assisted graphite halogenation followed by ultrasound-promoted exfoliation. Photographs of graphite chloride (b), a graphite chloride dispersion in DMF (c), and graphite bromide dispersion in DMF (d). Reproduced with permission from ref. [70]. Copyright 2012 Macmillan Publishers Ltd.

If all-carbon graphene has so far attracted enormous attention in mass media, the fact that polymerized organic crystals can also be exfoliated to create two-dimensional sheets should likewise open a new age of innovation in materials design. The subject is in its infancy, but two recent papers in Nature Chemistry will pave the way for these exciting 2D-polymers [72,73]. Both studies exploit the photopolymerization of single crystals, in particular anthracene-based monomers, where photochemical dimerization is facilitated by aromatic unit stacking. The resulting 2D-photopolymerized crystals can be exfoliated into sheets by dispersion in NMP at $50{ }^{\circ} \mathrm{C}$. Analyses by SEM and AFM (atomic force microscopy) reveal that multilayer materials were isolated in most cases, however a few monolayer sheets were also detected (Figure 11). As expected, sonication's role in exfoliation proved decisive, although the protocols are far from optimized. Short irradiation of NMP dispersions led to multilayer sheets, whereas full exfoliation to monolayers was only achieved by lengthy operations (1-2 weeks) that involved slow rotation in round-bottomed flasks or the use of a fluorinated acid as the intercalating agent. It should be noted that the exfoliation of covalent organic frameworks (COFs) and molecular organic frameworks (MOFs) is not new. However, the resulting sheets were not robust enough. In the present cases, the exploitation of covalent bonding in 2D-structures means that the sheets were able to support their own weight when aggregates were suspended across $\mu \mathrm{m}$-scale holes. These properties make these structures suitable for use in optoelectronics or artificial membranes. 

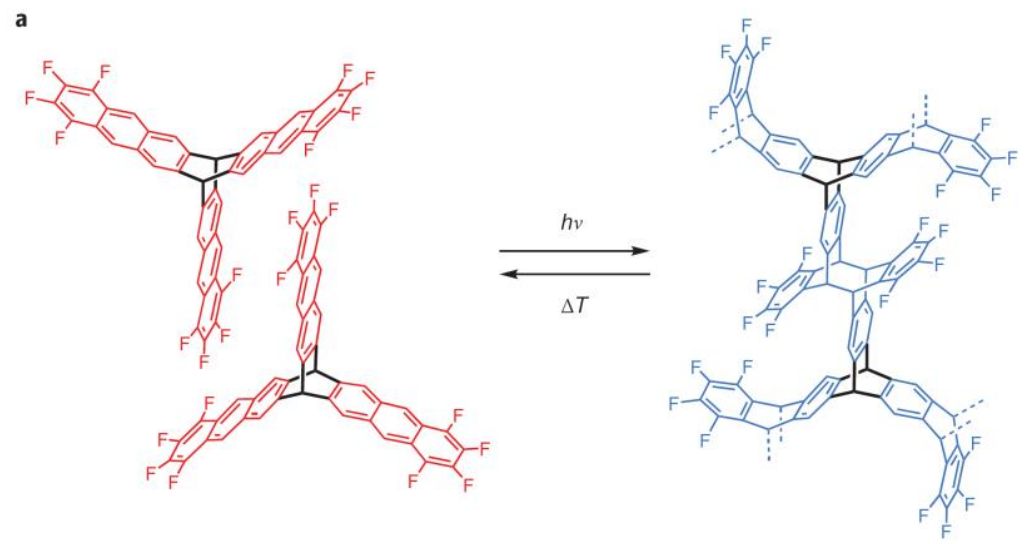

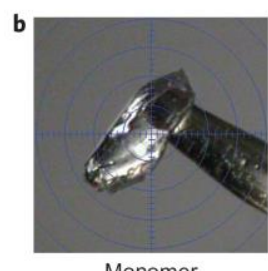

Monomer

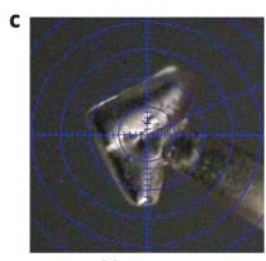

Monomer

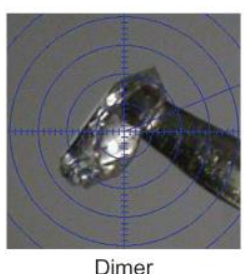

Dimer

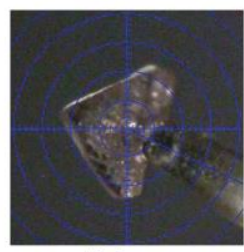

Polymer
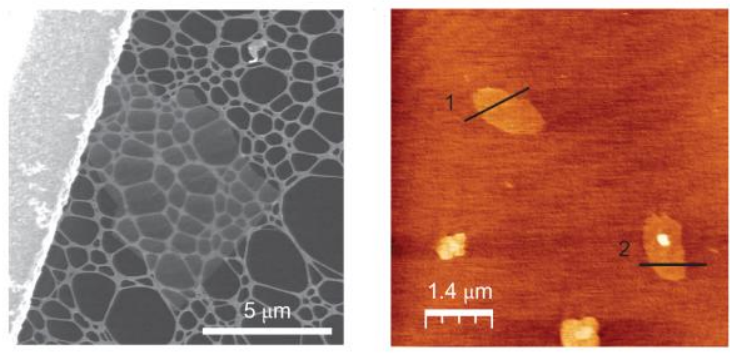

Fig. 11. (a) Chemical structure of a tetrafluoro-triptycene-based monomer (fantrip) and the corresponding 2D-polymer, poly(fantrip). (b) A single crystal of the monomer before and after irradiation $(223 \mathrm{~K}, 460 \mathrm{~nm}, 80 \mathrm{~min})$. (c) A single crystal of the monomer before and after irradiation $(223 \mathrm{~K}, 460 \mathrm{~nm}, 150 \mathrm{~min})$ followed by a second irradiation $(223 \mathrm{~K}$, $400 \mathrm{~nm}, 70 \mathrm{~min}$ ). The bottom panel shows SEM and AFM images of exfoliated sheets. Reproduced with permission from ref. [72]. Copyright 2014 Macmillan Publishers Ltd.

\subsection{Modification of Polymeric Structures}

The generation and properties of mechanically responsive polymers has most certainly become a hot topic in macromolecular science. Mechanical energy can be harnessed in multiple chemical transformations, such as mechanochromism, analyte detection and self-healing. The basic strategy relies upon the concept of the mechanophore; small structural units embedded into long chain polymers which undergo site-selective scission [6-8,12]. Although numerous solid-state and solution methods can be used to activate polymers [11], sonication holds the unique ability of providing shear forces and strain in solution by virtue of the mechanical events associated with cavitational collapse. A seminal study in the early 1980s reported the fast degradation of weak peroxide linkages along a polymer backbone under sonication (relative to control polymers) [74]. This was followed by more precise explorations by Moore and coworkers showing how azo or benzocyclobutene mechanophores, approximately installed in the 
centre of a polymer chain and subjected to ultrasonic irradiation, underwent site-specific cleavage [75,76]. The latter was particularly appealing to modern literature, not only because it showed the accelerating effect of ultrasound-induced force, but also because it showed reaction pathways that challenge the current Woodward-Hoffmann rules based interpretation. Thus, both cis- and trans-functionalized benzocyclobutenes undergo ring opening leading to the E,E-configured diene, a result that was further corroborated by derivatization with an appropriate chromophore (Figure 12). This finding is unexpected from a thermal activation point of view (only the trans isomer should afford the E,Ediene while the cis isomer is expected to give the E,Z-diene). Alternatively, a photochemical mechanism would give rise the reverse result (cis and trans isomers leading to $E, E$ and $E, Z$-dienes, respectively).
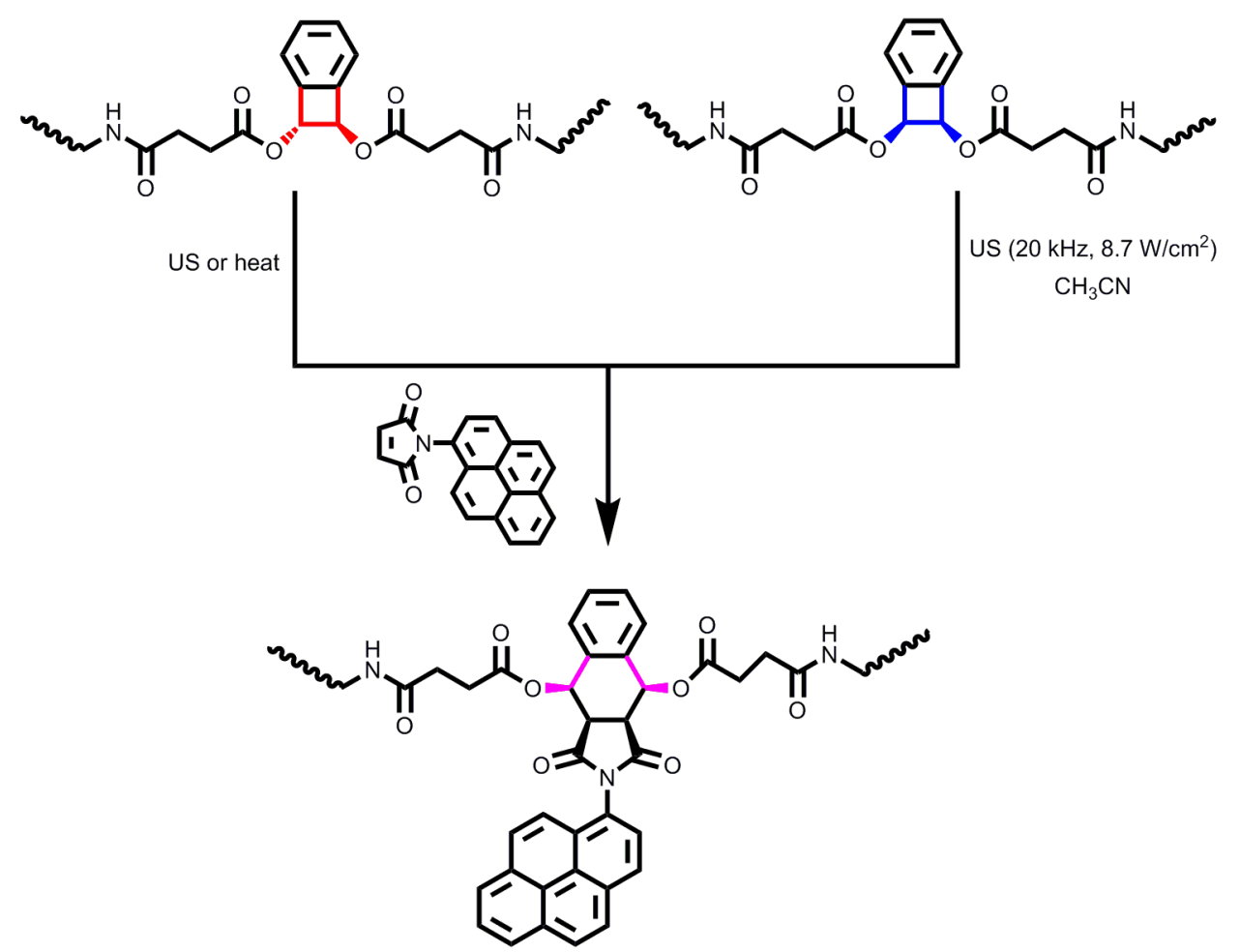

Fig. 12. Ultrasound-induced ring-opening transformations of benzocyclobutene mechanophores installed in polymer chains.

This "nonclassical" electrocyclic ring-opening reaction, along with other striking results that are markedly different from what is expected from thermochemical pathways, clearly require an extension of theoretical models to mechanical forces and distortioninduced instabilities $[18,77,78]$. This will not be discussed herein, although a few, merely qualitative, conclusions should be mentioned: a) mechanical scission caused by strain after bubble collapse is most likely responsible for polymer cleavage; b) radical 
production has a negligible effect; c) thermally forbidden mechanisms may become mechanically allowed pathways within a certain range of forces.

If one assumes that, at least intuitively, sonication offers efficient mixing in liquids where forces are generated by turbulent regimes and elongational flows, this framework may be invoked in tracking mechanophore evolution [10]. There are actually some similarities between ultrasonic and transient elongational flow degradation of polymers, such as the propensity for midchain cleavage and molecular weight dependence. There should be some resemblance between the adiabatic compression triggered by bubble generation as ultrasound propagates and transient elongational flow, which is obtained by forcing a liquid across a constriction. As a result, a high velocity gradient is created. However, some divergences exist between the hydrodynamic types and these have been discussed in detail by Nguyen et al. as they evaluate the kinetics of ultrasonic degradation for dilute solutions of polystyrene in decalin [79]. Firstly, the cavity is filled with a gas that undergoes constant compression and expansion cycles, and secondly acoustic pressure varies along the bulk medium as a function of time. Accordingly, the strain rate changes at various time intervals during bubble collapse. As viewed in Figure 13, the temporal evolution of strain rates depends on the radius of the collapsing bubble. The latter is created above an intensity threshold and will become unstable enough after reaching a critical diameter.

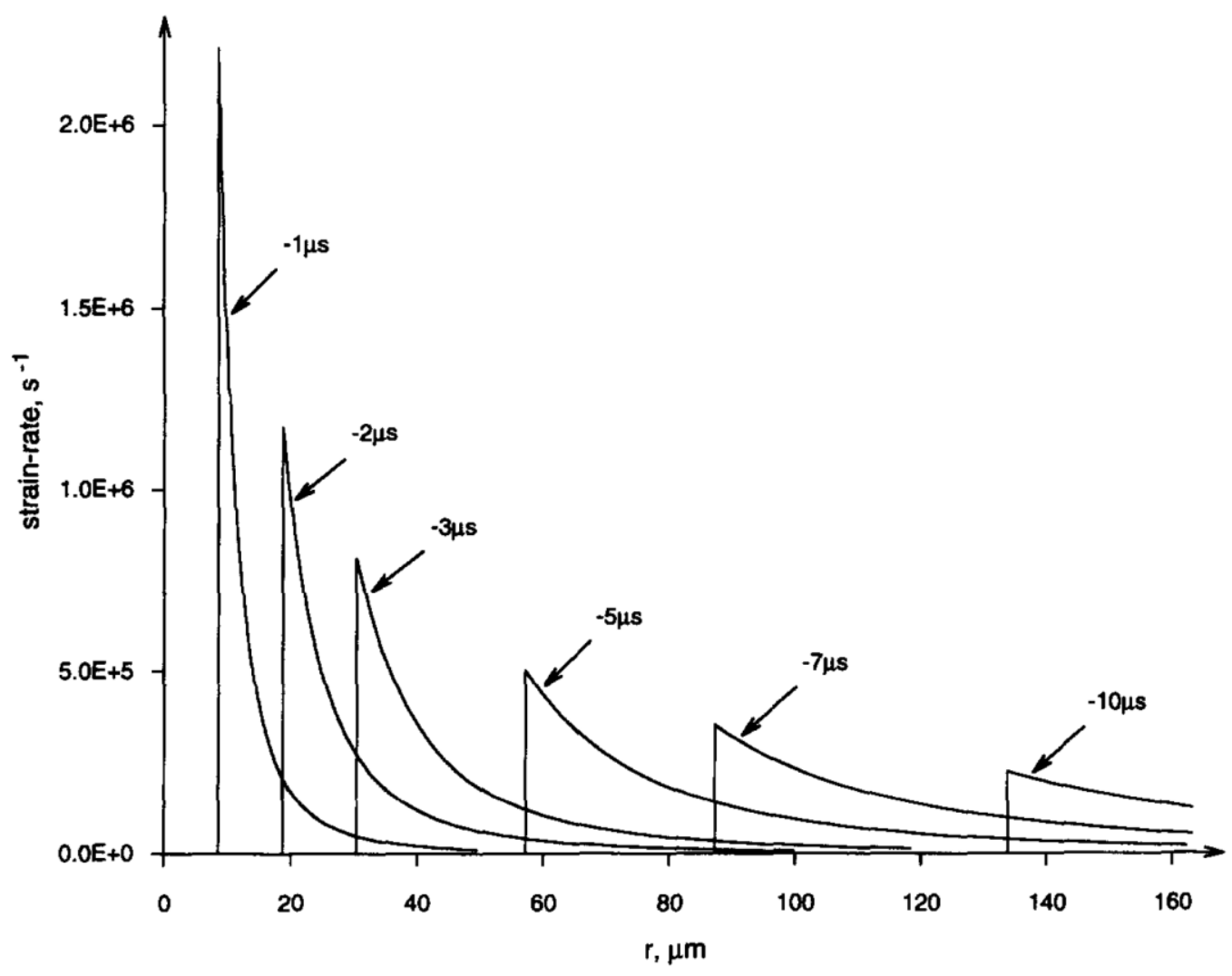


Fig. 13. Strain rate distribution during bubble collapse versus various implosion times. The distance from the cavity centre $(r)$ is given in $\mu \mathrm{m}$ and full collapse occurs at $t=0$. Reproduced with permission from ref. [79]. Copyright 1997 Elsevier Science Ltd.

Unlike transient elongational flow, the flow induced by sonication at the collapse stage is time dependent. This means that the important factor in kinetics modelling is not spatial distribution, but temporal variation in the fluid strain rate [79]. In an ultrasonic experiment, both the limiting molecular weight $\left(M_{\text {lim }}\right.$, i.e. the molecular weight below which no chain scission takes place regardless of the degradation time) and the degradation rate are dictated by an effective strain rate ( $\left.\dot{\mathrm{e}}_{\mathrm{eff}}\right)$, which prevails during bubble collapse. By extrapolating the kinetics results obtained in transient elongational flow to ultrasonic degradation, and bearing in mind the $M_{\mathrm{lim}}$ values determined in sonication experiments, Nguyen and coworkers concluded that éff should be $\geq 3 \times 10^{6} \mathrm{~s}^{-1}$ (Figure 14). The curve suggests that a polymer chain near the cavitating bubble undergoes a range of strain rates and therefore multiple fragmentations may occur if the daughter chains are larger than $M_{\text {lim }}$. Similar behaviour can be inferred from transient elongational flow experiments, as midchain scission is the dominant process at low strain rates. At higher strain rates, however, shorter molecular chains appear in solution and the resulting molecular weight distribution of the degraded polymer will be close to the distributions obtained by cavitational flow [79].

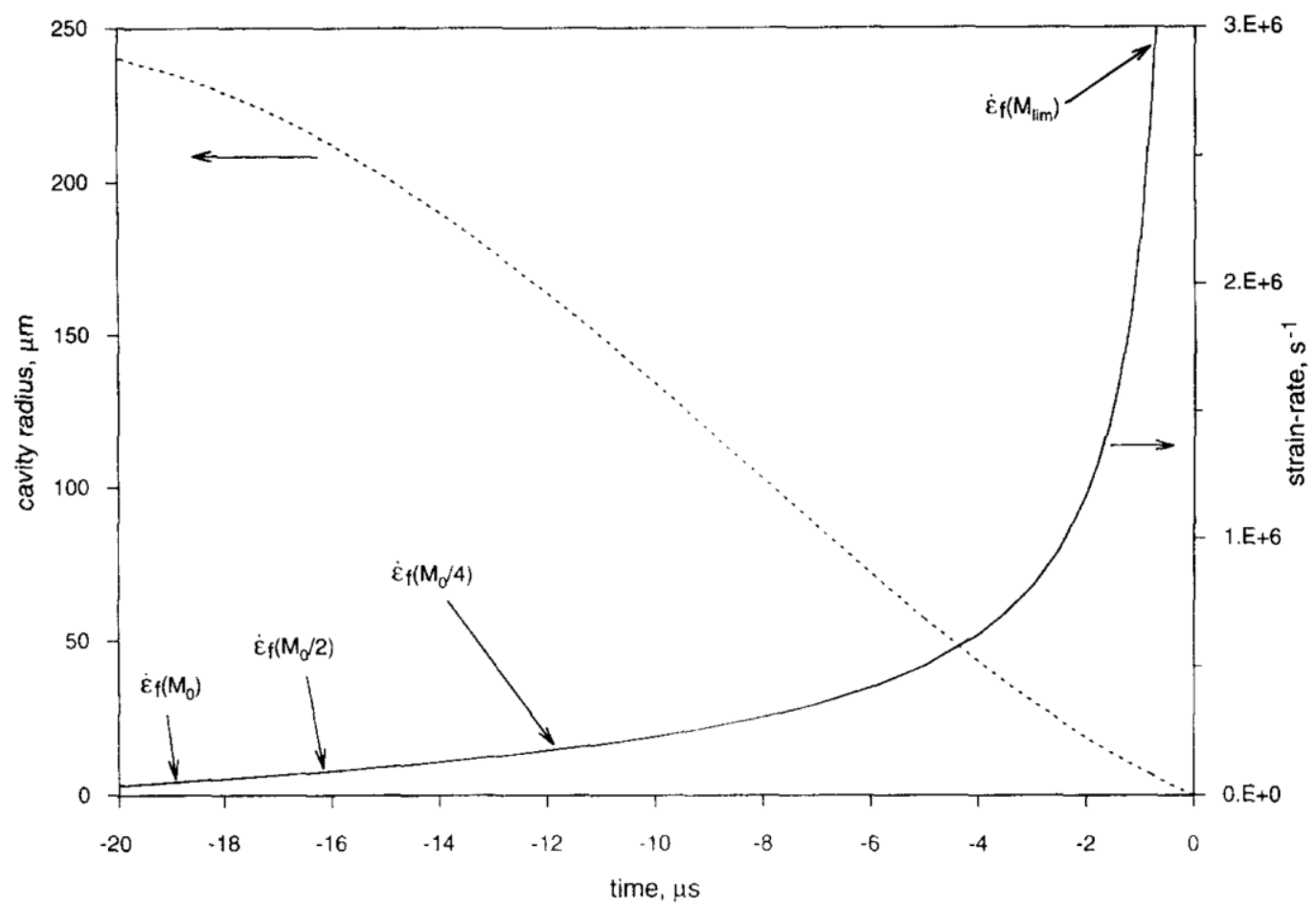


Fig. 14. Temporal evolution of the bubble radius (left axis, in $\mu \mathrm{m}$ ) and strain rate maximum (right axis, in $\mathrm{s}^{-1}$ ) during bubble implosion. Reproduced with permission from ref. [79]. Copyright 1997 Elsevier Science Ltd.

As stated previously, the advancements in controlled polymer scission via sonomechanochemistry are remarkable with new exploration occurring at great pace. Whatever the actual mechanism may be, recent synthetic schemes focus on new functional groups and applications. Thus, a dioxetane mechanophore inserted into a polymer chain represents another weak linkage that can be selectively cleaved under sonication [80]. Blue luminescence, whose intensity and colour was modified by the presence of energy-transfer acceptors, was observed from the resulting excited ketone species (Figure 15). This protocol also constitutes a valuable step towards detecting failures or structural defects in polymeric materials. Moore and associates have also introduced a biomimetic-like material with reversible responses in the form of a metastable poly(ortho-phthalaldehyde) that undergoes ultrasound-induced mechanical depolymerization to its components via a heterolytic mechanism. Repolymerization can subsequently be achieved by a chemical initiator [81]. It is worth pointing out that repeatable force-induced cycles which incorporate flex-activated mechanophores (serving as crosslinkers) into elastomeric networks have recently been demonstrated. Activation takes place by applying compressive stress, thus releasing a small molecule that diffuses out of the polymer matrix $[82,83]$.

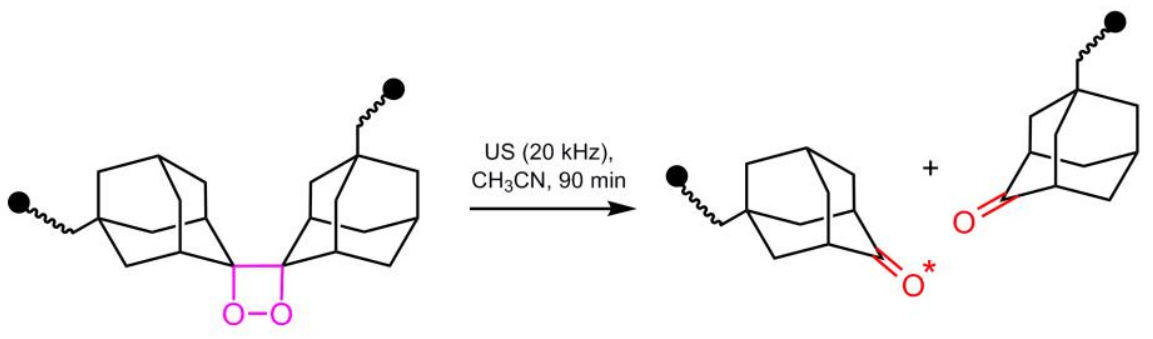

Onun poly(methyl acrylate) polymer 


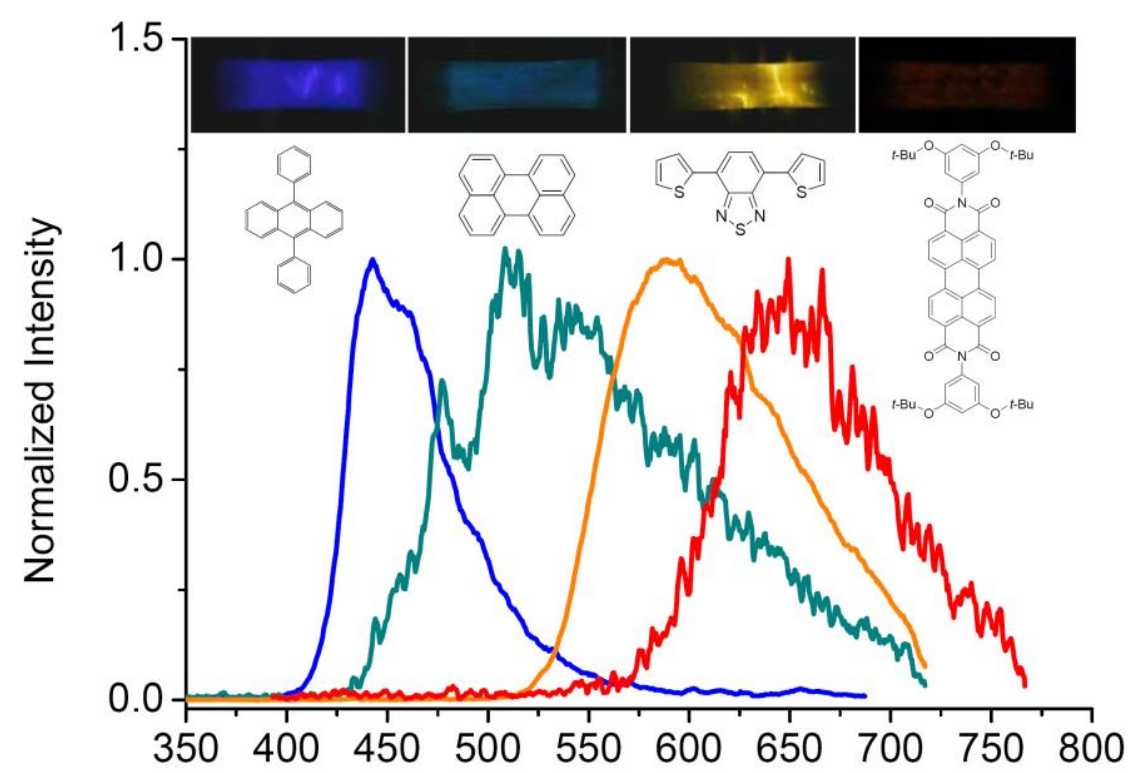

Fig. 15. Top: Sono-scission of a polymer chain which includes a 1,2-dioxetane mechanophore to form an adamantanone-excited species with blue light emission. Bottom: Luminescence spectra obtained during the mechanical stretching of polymers containing organic acceptors show different colours and intensities. Reproduced with permission from ref. [80]. Copyright 2012 Macmillan Publishers Ltd.

A new productive chemical change was also observed when mechanical stress caused by ultrasonic irradiation was applied to triple bonds centred on a poly(methyl acrylate) backbone [84]. Further reaction with azides gives rise to an isoquinoline derivative, which points to transoidal bending activation that deviates from the expected product (1,2,3-triazole) observed for Huisgen-type cycloadditions of azides with cisoidal triple bond arrangements in strained alkynes. Once again, this result is outstanding and illustrates how mechanochemistry can do things which are otherwise difficult or impossible under conventional conditions. Nevertheless, site-selective polymer scission under mechanical stress should neither be a universal trend nor a chemical panacea. A retro-click reaction that has caused enormous excitement in the chemical community, given the wide applicability of the bio-orthogonal alkyne-azide ligation, might not be genuine; an editorial expression of concern has been published and the subject is under confidential investigation [85]. Nevertheless, as rightly pointed out by experts in the field, these circumstances will have consequences for specific transformations and do not weaken the principle message conveyed by polymer mechanochemistry which has been based on numerous and testable examples [86]. Thus, a recent application to surface functionalization should be mentioned in the present context. Silica nanoparticles $\left(\mathrm{SiO}_{2}-\right.$ 
NPs) grafted with a polymer chain containing a maleimide-anthracene cycloadduct were also subjected to ultrasonication and afforded mechanophore scission via a retro-[4+2] cycloaddition. The mechanophore-anchored polymer-grafted $\mathrm{SiO}_{2}-\mathrm{NPs}$ were prepared by standard protocols (immobilization of triethoxysilane-terminated monomer initiator to silica particles followed by surface-based living radical polymerization). As expected, sonication was able to generate elongational flow after bubble collapse, thereby transducing mechanical force into the polymer chains. The weakest point should be the polymer- $\mathrm{SiO}_{2}-\mathrm{NP}$ heterointerface, which underwent preferential cleavage. Polymer chains of various molecular weights linked to $\mathrm{SiO}_{2}$-NPs were also tested. Results evidenced a linear response to polymer chain sizes plus a threshold molecular weight dependence similar to that of homopolymers. Moreover, mechanophore scission altered the morphology of the grafted $\mathrm{SiO}_{2}$-NPs, which exhibited irregular patterns, unlike hexagonal arrangements before sonication [87].

Mechanochemical transduction aided by sonication can also be achieved at a supramolecular level. Given the dynamic character (self-assembly and disassembly) of non-covalent interactions, reversible transformations to create new responsive polymers may potentially be accomplished. As shown in Figure 16, a cross-linked network containing a europium(III) salt undergoes metal-ligand dissociation upon exposure to pulsed ultrasound in solution. This disassembly is only possible with a high-molecular weight supramolecular entity, thus again showing the same size dependence as reported for covalent polymer mechanophores. The strength of the metal-ligand interaction is also critical, as polymers that contain more strongly coordinating ligands do not dissociate under irradiation [88]. Interestingly, the authors searched for mechanically healable responses and, to this end, polymer films were cut into pieces, gently pressed and exposed to ultrasound, while either directly immersed in a $\mathrm{CH}_{3} \mathrm{CN}$ solution or in a sealed bag. In both cases, piece welding was observed and the original mechanical properties of the material were fully restored by this ultrasonic mending protocol. 


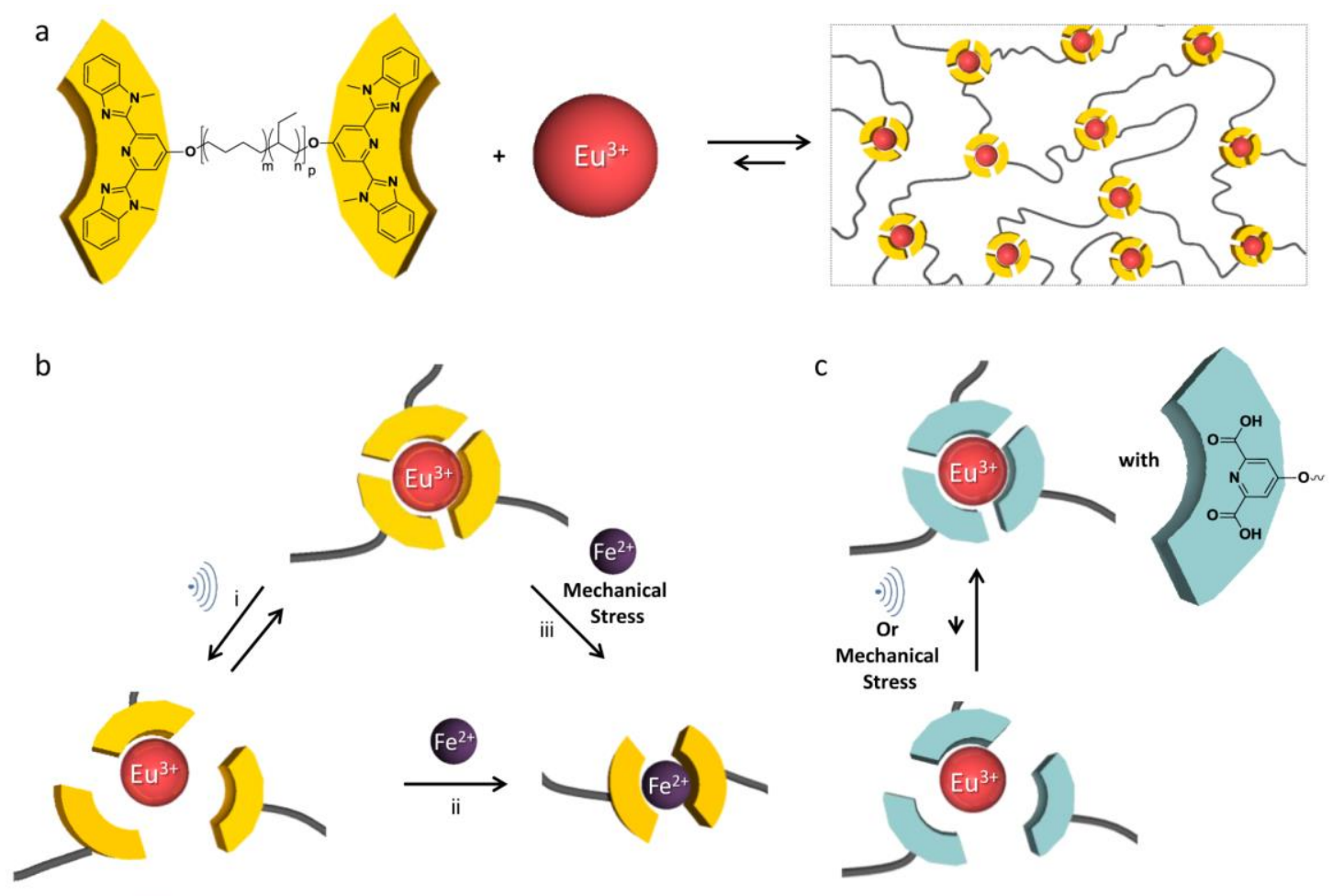

Fig. 16. Mechanochemical evolution of metallosupramolecular polymers generated by (a) combination of a $\mathrm{Eu}(\mathrm{III})$ salt and a telechelic poly(ethylene-co-butylene) with 2,6-bis(1'methylbenzimidazolyl)pyridine ligands at the termini; counterions are omitted for clarity. (b) Reversible dissociation upon ultrasonication (i); irreversible metal exchange with $\mathrm{Fe}$ (II) ions as a result of (ii) ultrasonication or (iii) other mechanical forces. (c) Dipicolinic acid ligands bind strongly to Eu(III) and the supramolecular network cannot easily be disassembled under mechanical stress. Reproduced with permission from ref. [88]. Copyright 2014 American Chemical Society.

Aida and coworkers have investigated the influence of low-frequency, audible range sound on macromolecular alignment. The group synthesized a zinc porphyrin that undergoes self-assembly via coordination and hydrogen bonding interactions. The resulting supramolecular nanofibre preferentially aligns itself parallel to the propagation of audible sound (Figure 17), a fact that can be visualized by linear dichroism (LD) spectroscopy [89]. Observations point to an alignment of the nanofibres flowing around the sidewall of the cuvette and them becoming parallelly oriented to the direction of liquid vibration. Since a large hydrodynamic gradient (i.e. velocity gradient) should occur at the boundary layer of the liquid flowing near the wall surface, a large LD intensity could also be recorded at the sidewall. The phenomenon appears to be quite general for linear nanofibres, while dendritic zinc porphyrins exhibit a similar effect at higher concentrations. On the other hand, no acoustic LD responses were detected for rod- 
shaped structures. The protocol may find potential applications in vibration sensing technologies.
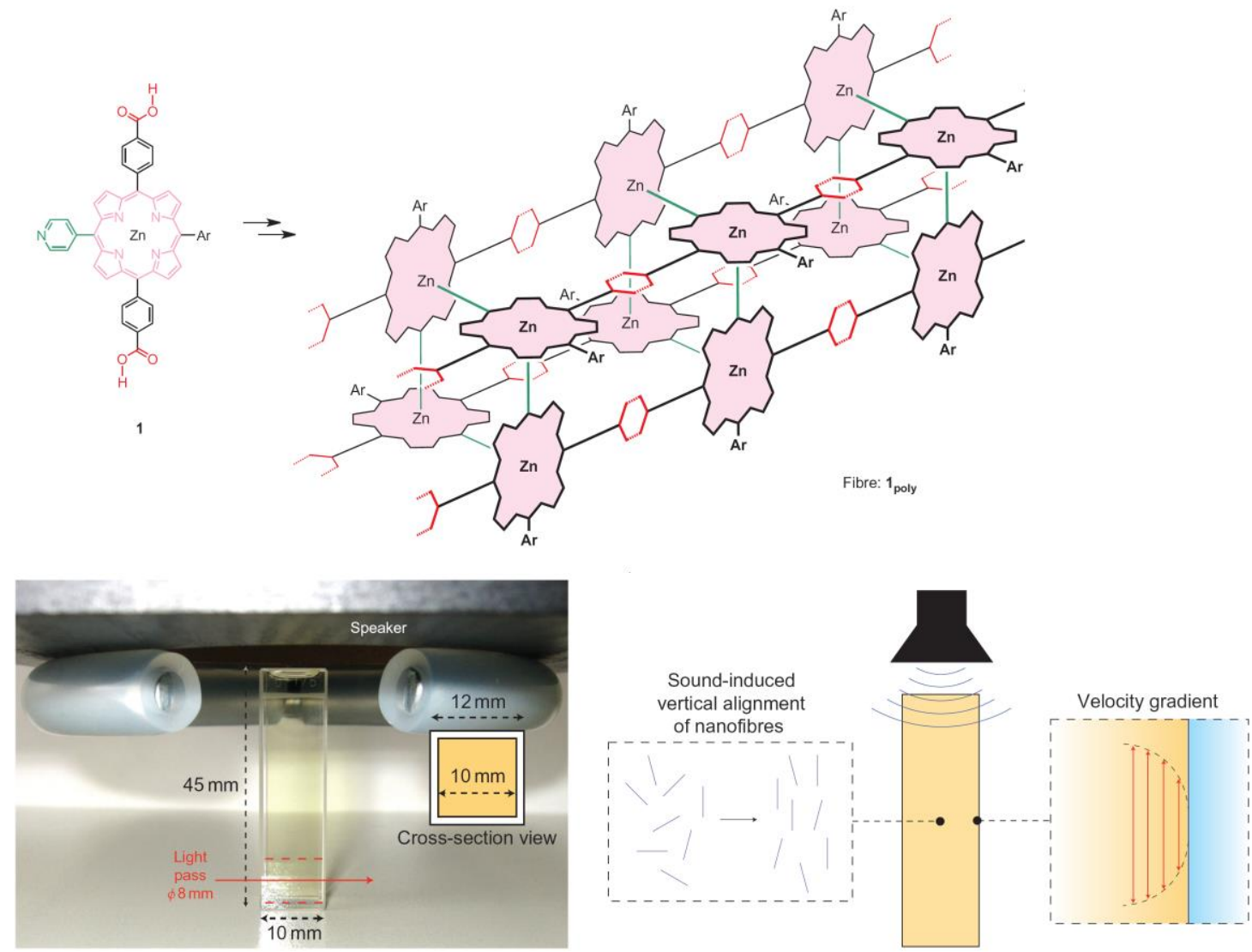

Fig. 17. Top: self-assembled zinc porphyrin leading to supramolecular nanofibres. Bottom: the designed nanofibres can be acoustically aligned in solution with audible sound emitted from a loudspeaker located $20 \mathrm{~mm}$ above the cuvette. Linearly polarized light was used to record LD spectroscopy. Reproduced with permission from ref. [89]. Copyright 2010 Macmillan Publishers Ltd.

In line with the preceding example, hydrodynamic orientation has also been detected in molecules that are firstly subjected to light and then exposed to audible sound capable of eliciting macromolecular changes. Thus, a linear molecule containing a central azobenzene moiety and three long alkyl chains at each end self-aggregates into supramolecular nanofibres when the azo group is trans-configured. Photochemical irradiation (UV light) causes its isomerisation into the cis-azobenzene derivative, which leads to amorphous aggregates (Figure 18) [90]. Notably, the assembly process was highly dependent on the length of the alkyl chains and only a 12-carbon chain gave rise to aggregation from the trans-isomer. 6-, 12- and 16-carbon chains aggregated in an amorphous fashion in the $c i s$-isomer. LD spectroscopy showed no preferential orientation in solution. However, when samples were irradiated with audible sound, the nanofibres 
were found to align with each other, whereas no change was detected in the amorphous cis-aggregates.

(a)
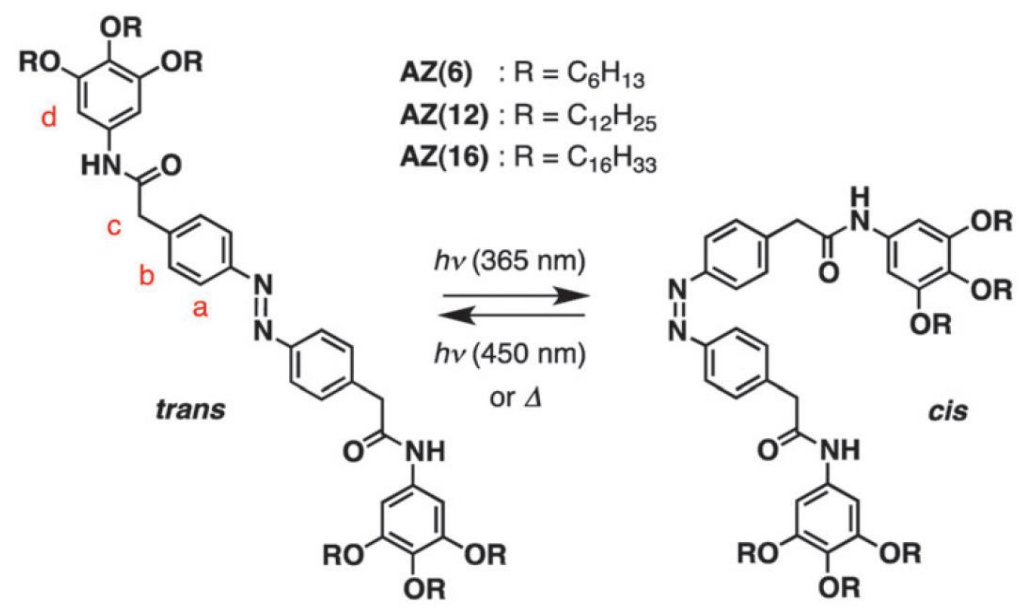

(b)
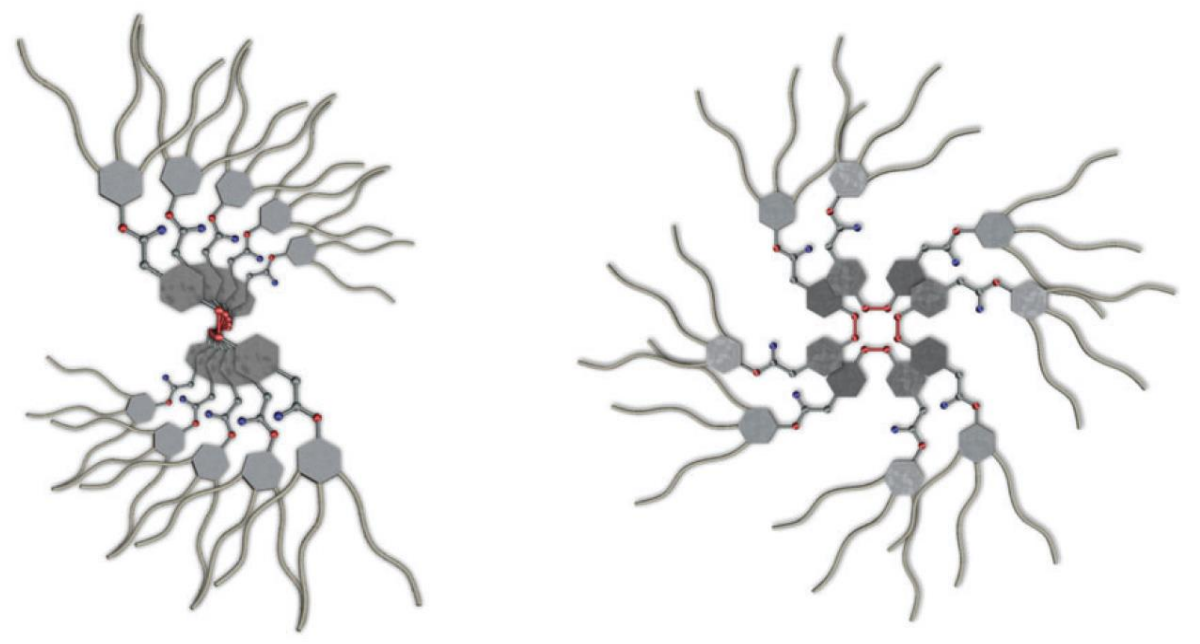

Fig. 18. (a) Chemical structures of trans- and cis-azobenzene derivatives which undergo reversible photoisomerization. (b) Putative self-assemblies; only nanofibres generated from the trans-isomer aligned in a sound-induced fluid flow. Reproduced with permission from ref. [90]. Copyright 2014 The Royal Society of Chemistry.

\section{Mechanical Action on Biostructures}

The effects of sound waves on biological tissues and structures have long been known. In general, prolonged sonication will be detrimental, lead to extensive cellular damage and chemical biomolecule modification via cavitation processes. Ultrasonic biophysics deals with the study of mechanisms which account for the interaction of ultrasound and biological materials [91]. This damage can however be harnessed for specific therapeutic applications [92,93]. Furthermore, the changes experienced by sound 
waves in biological media can also be viewed as the basis for diagnostic ultrasound and biomedical imaging [94,95]. In particular, drug delivery may be greatly enhanced under sonication. Shock waves induce mechanical forces that transiently open polymeric micelles and polymersomes, thus releasing their contents. This mechanism is well accepted (vide infra), although the effects of ultrasound on cell apoptosis or genotoxicity must still be elucidated [96]. Likewise, copolymer micelles which are a suitable, FDAapproved vehicle for drug delivery can be functionalized to respond to multiple stimuli. For instance, the incorporation into the micelle of a mechanophore that can be selectively cleaved under ultrasound plus the inclusion of a disulfide bond which is sensitive to the reducing action of glutathione illustrates this aspect [97].

It is generally considered that ultrasound waves change the permeability properties of liposome membranes due to transient cavitation, where the collapse of air bubbles near the lipid membrane creates pores that alter the orientation and hydrophobicity of the lipid structure [98]. Drug delivery via acoustically sensitive liposomes is also largely influenced by the content and structure of the lipid systems. Drug transport can be facilitated by means of lipid molecules, within the liposome membrane, which are capable of creating local instabilities. While such instabilities do not disturb membrane integrity before sonication, they respond once the acoustic stimulus is applied and then heal when the irradiation is turned off [99]. Thus, at appropriate phosphatidylethanolamine (PE) phospholipid levels, the membrane displays a typically lamellar structure with zero intrinsic curvature $(\mathrm{H}=0)$ that undergoes topological changes after exposure to ultrasound and converts into a non-lamellar structure, with negative intrinsic curvature $(\mathrm{H}<0)$, forming pores through which the drug can readily be released (Figure 19).
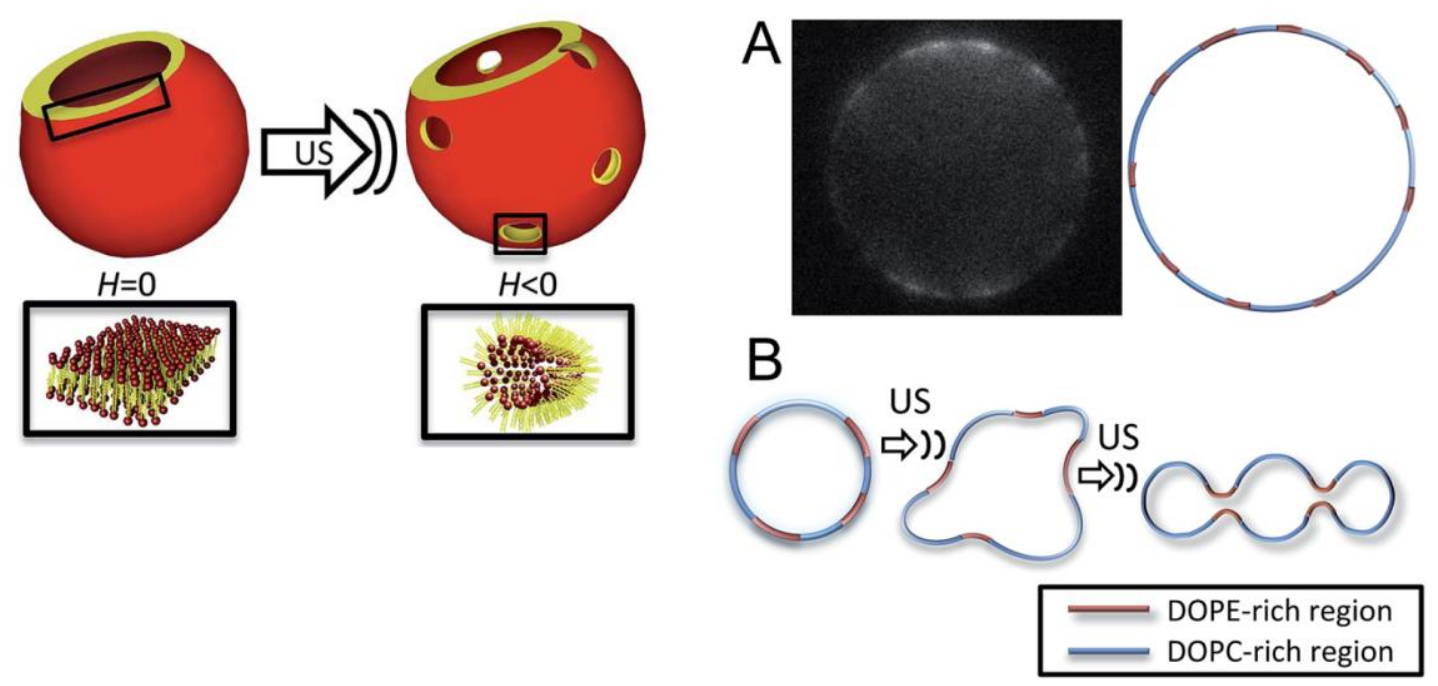
Fig. 19. Left: schematic representation of the proposed mechanism for topological changes in dioleoyl phosphoethanolamine (DOPE) based liposomal membranes upon ultrasound irradiation. Right: (A) a giant DOPE-based unilamellar vesicle, before sonication, which shows an inhomogeneous membrane; DOPE-rich domains of negative curvature are marked in red, embedded in zones rich in dioleoyl phosphocholine (DOPC) of zero mean curvature. (B): Illustration of shape changes upon ultrasound stimuli. Reproduced with permission from ref. [99]. Copyright 2014 The Royal Society of Chemistry.

A recent simulation using molecular dynamics has evaluated the creation of pores in lipid bilayer membranes by inducing shock waves in a system containing an array of nanobubbles next to those membranes [100]. This model shows that the extent of damage depends on the orientation of the bubbles relative to the shock wave direction as well as the distance between the bubbles. The argument may, in principle, be extrapolated to the context of cavitation-induced microbubbles. However, the authors used a simplified bubble system that did not contain any water vapour molecules, but did include specific bubble alignment to the membrane. Simulations thus point to temporary lipid bilayer disruption under such conditions. This disruption would then be recovered after the damage, which contrasts with the permanent alteration that cavitation creates in solid surfaces.

An indirect and clever method for inducing drug release is the inclusion, inside the liposomes, of magnetic nanoparticles which can be activated by a pulsating magnetic field that triggers the generation of ultrasonic vibrations $(\sim 30 \mathrm{kHz})$. Proof of concept lies in the release of molecules, such as $\mathrm{MgSO}_{4}$ and 5(6)-carboxyfluorescein, being accomplished from magneto liposomes loaded with $\mathrm{Fe}_{3} \mathrm{O}_{4}$ or FePt nanoparticles, both in homogeneous and inhomogeneous magnetic fields [101].

More productive chemical results, which still harness the destructive action of ultrasound on certain bonds, can be attained when sonication is applied to biological fluids (e.g. protein solutions) en route to bionanomaterials [15]. A conspicuous example can be found in sonochemically-prepared protein microspheres, in which the interplay of mechanical effects (emulsification) and chemical effects (formation of transient species) is noticeable. A protein emulsion is readily created at the interface between two immiscible liquid phases, while radicals generated by water sonolysis promote disulfide bond cross-linking between cysteine residues. Surface modifications, via conjugation with monoclonal antibodies or RGD-containing peptides, can also be carried out $[102,103]$. The sonochemical preparation of chitosan microspheres also exploits the intermolecular cross-linking of imine bonds from the sugar precursor [104].

Cell disruption under the action of sound waves appears to be of particular benefit in the case of biofilm elimination. A bacterial biofilm is a microbial community attached 
to a substrate or interface and which is also embedded in a matrix of polymeric substances generated by such bacteria. Biofilms are a major problem in prostheses as opportunistic pathogens (e.g. Escherichia coli, Staphylococcus aureus or Staphylococcus epidermidis) develop rapidly after surgery. Ultrasound has been frequently used as an effective tool for biofilm removal. Surprisingly, literature also describes an antagonistic effect, i.e. bacterial viability may be enhanced under sonication. The subject has been recently reviewed and it was found that both effects depend on factors such as frequency, intensity, materials used for ultrasound diffusion, the presence or absence of cavitation, as well as the type of bacteria [105]. The combined use of therapeutic ultrasound with antibiotics usually decreases bacterial viability in vitro and in vivo [106]. On the other hand, sonication at high frequency is unable to kill bacteria, though some damage to the surrounding tissue is produced [107].

Cavitational effects on cells and bacteria can be assessed by culturing a specific strain into a container that is then exposed to cavitation generated by ultrasound, shock waves or a laser. The so-called Harvey chamber was a pioneering model with which to evaluate the biophysical effect of ultrasound. Here, the pressure values from the impact of a liquid jet produced during bubble collapse and the accompanying shock wave, are compared against the maximum stress the cell surface can withstand before rupture [108]. Damage to microbial cells and degradation of their polymer chains also reflect the shear stress triggered around oscillating or collapsing microbubbles. Thus, the rate of intercellular protein release from yeast cells has been used to quantify the mechanical effects of ultrasound. Yeast cells are relatively rigid and fragmentation only occurs in the vicinity of cavitation bubbles, while microstreaming is largely inefficient in fragmenting [109]. Figure 20 shows a merely qualitative relationship between forces in the ultrasonic field and the strength of different classes of biological cells. The frequency effect of cavitation is indicated by the amount of protein released at $20 \mathrm{kHz}$ and $130 \mathrm{kHz}$. The frequency effect under microstreaming is based on the boundary layer, of thickness $\delta$, separating the inner and outer streaming vortices:

$\delta=(2 \eta / \omega \rho)^{1 / 2}$

where $\eta$ is the shear viscosity, $\omega$ the angular frequency and $\rho$ the density of the liquid. The boundary layer has a thickness of about $4 \mu \mathrm{m}$ at $20 \mathrm{kHz}$ and $0.56 \mu \mathrm{m}$ at $1 \mathrm{MHz}$ in water. The smaller size of the layer at high frequencies results in high velocity gradients and shearing stress of $c a$. $550 \mathrm{~Pa}$. Bubbles vibrating at $20 \mathrm{kHz}$ as source of microstreaming are capable of breaking erythrocites which leak haemoglobin when shear stress exceeds $450 \mathrm{~Pa}$ [109] (Figure 20). 


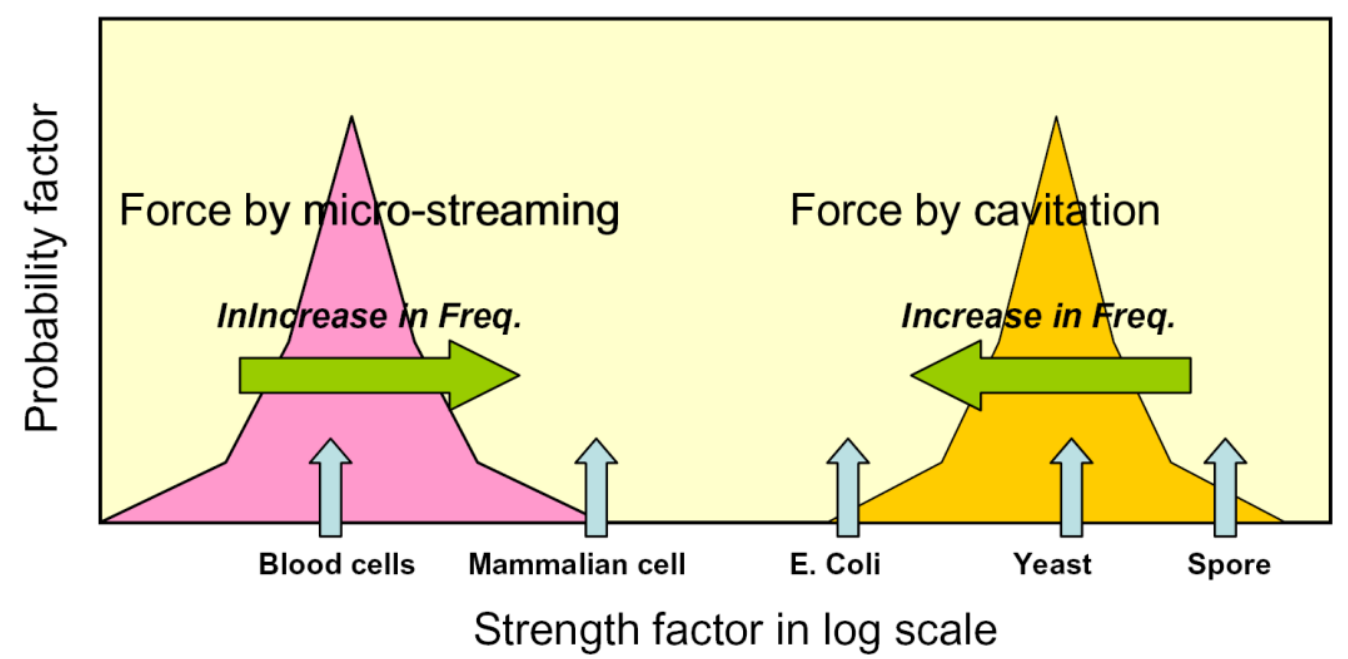

Fig. 20. Schematic diagram showing the relationship between cavitational forces and cell surface strength. Rigid structures are broken by bubble collapse, with little or no microstreaming effect. The $x$-axis (strength factor; no numerical values are given) provides an indication on the shear forces required to disrupt mammalian or microbial cells. Reproduced with permission from ref. [109]. Copyright 2008 Elsevier Science Ltd.

Similar conclusions on the negligible effect microstreaming has on biofilm disruption have been reached by Fernandez Rivas et al. using a small-scale ultrasonic device with the ability of locally remove layers of metals, polymers or biomaterials from a glass slide (Figure 21) [110,111]. The innovative idea here is the use of a silicon surface containing micropits where individual gas bubbles can be entrapped. Ultrasonic vibration gives rise not only to acoustic streaming from the oscillating surface, but also results in gas bubble pinch-off from the micropits at high enough pressures. The glass cavitation chamber ( $25 \mathrm{~mm}$ outer diameter and $6 \mathrm{~mm}$ depth) is glued to a piezo element working at a frequency of $200 \pm 5 \mathrm{kHz}$ at the bottom. The biofilm was removed rapidly when the bubble cloud was attracted by the biofilm-covered substrate (Figure 21). However, no removal was observed by streaming alone or by bubble clusters not attracted to the glass surface. This behaviour was also observed for a hydrogel mimicking biofilm characteristics. 

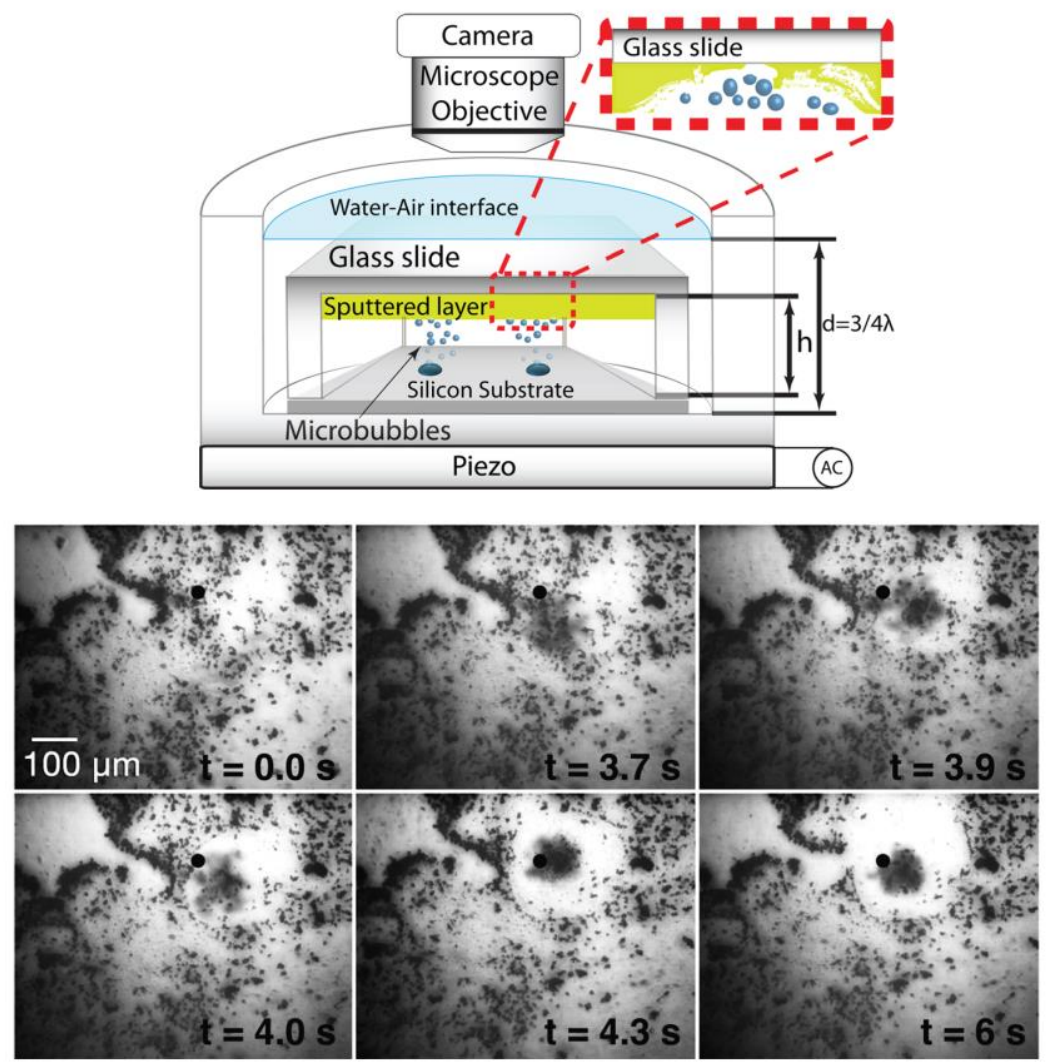

Fig. 21. Top: schematic setup of an ultrasonically vibrating micropitted silicon surface. The cavitation chamber is filled with pure liquids or a cell cultivation liquid for biological assays. Bottom: temporal recording of a biofilm removed by microbubbles. The gray area with black dots is the zone covered by biofilm. The pit is indicated with a large black dot. Microbubbles can be identified as the blurred dark region surrounding the pit. Reproduced with permission from ref. [110]. Copyright 2012 AIP Publishing.

The increasing growth of neuropathologies, especially in Western countries, that are associated with prions and other aggregates of misfolded proteins has become a serious concern in biomedical research. Amyloid fibril formation, for instance, appears to be the critical step in Alzheimer's disease. Ultrasound has been used to evaluate both the formation and disruption of amyloids, although contradictory results may be found as sonication parameters are often overlooked, much like in biofilms.

Sonication-induced fibrils, generated in thermostated baths at short irradiation times (ca. $1 \mathrm{~min}$ ), cause the formation of subsequent fibrils (self-seeding). The process is $\mathrm{pH}$-dependent and AFM images indicate that fibrils with diameters of more than $7 \mathrm{~nm}$ are obtained at $\mathrm{pH} 7.0$, which are thicker than those formed at $\mathrm{pH} 2.5$ [112]. Fibril breakage occurs at longer irradiation times, although ultrasound also produces a uniform fibril length distribution. A recycling model has been proposed as the fibrils in the sonicated 
samples markedly increase their length when left undisturbed [113]. The homogeneous size achieved under sonication bears some resemblance to the pluses associated with sonocrystallization, thereby favouring the nucleation step. The growth phase proceeds in a template-like manner by incorporating monomers into the ends of seed fibrils. Without sonication, the energy landscape is broad and additional break down or re-agglomeration mechanisms would result in a wide range of fibril lengths. The elongational field triggered by sonication modulates the free energy profile and leads to monodispersed fibrils with uniform size, as shown in Figure 22 (average molecular weight of 1,660,000 daltons or 140 mer) [114].

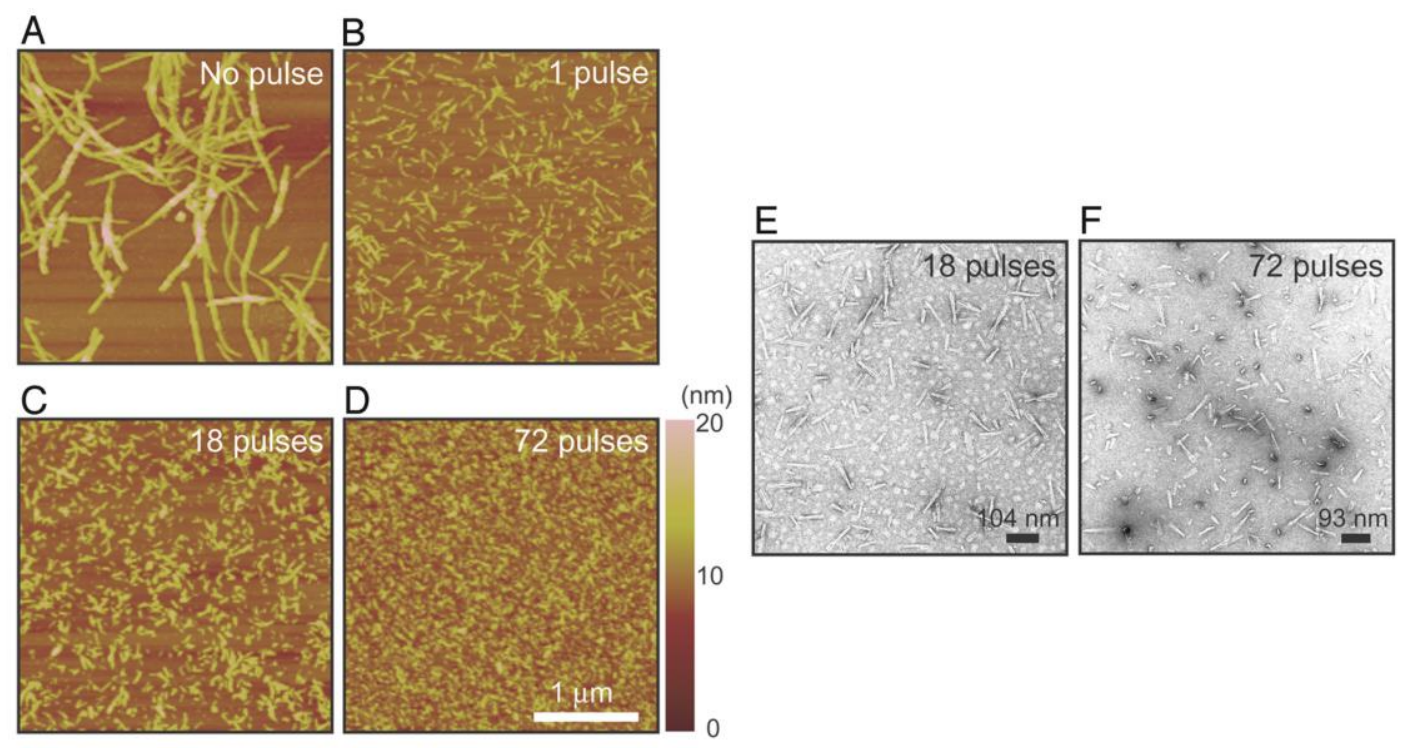

Fig. 22. Effects of pulsed ultrasound on the fragmentation of $\beta_{2}$-microglobulin. A-D show AFM images of the fibrils before sonication and after 1, 18 and 72 pulses, respectively. E-F show the corresponding electron microscopy images. Pulses were applied for 1 min with variable quiescent periods. Sonication was run at $37^{\circ} \mathrm{C}$ in a bath (ca. 17-20 kHz). Reproduced with permission from ref. [114]. Copyright 2009 American Association for the Advancement of Science.

Molecular dynamics simulations have recently been performed to ascertain the disruption of amyloid fibrils, which sheds light onto the cavitational event [115]. No significant changes in the peptide structure are observed at positive pressures. At negative pressures, however, a bubble is created, mainly around the hydrophobic residues of the transmembrane region. Most $\beta$-sheet structures are maintained even in the bubble, although the latter collapses after reaching a positive pressure and water molecules impact against the hydrophilic residues in the non-transmembrane region and disrupt the amyloid. Shorter amyloids require longer irradiation times for disruption to occur, because the number of hydrophobic residues is insufficient to serve as bubble nuclei, 
which is consistent with the above-mentioned experimental results. These simulations however, assume that bubbles are generated from water vapour and do not take into account the formation of microbubbles from existing gases in solution. Likewise, fragmentation induced by shearing forces was not considered either. Further molecular modelling reveals that force-assisted fragmentation and fracture times in amyloids depend on polymorphic substructures [116]. Also, fibrillation appears to be strongly dependent on chemical composition as proven by another study into chitin derivatives under ultrasonication and gas bubbling in water [117].

\section{Ultrasonically-driven Motion}

If any aspect of ultrasonic irradiation is tightly linked to molecular biology, it must surely be mechanotransduction, by which mechanical input is harnessed for autonomous motion or converted into (bio)chemical signals. Although the morphologies of living organisms are encoded in their genomes, cells are otherwise active gels and the evolutionary processes that lead ultimately to shape are rooted in mechanical forces. The emerging field of mechanobiology aims to understand how mechanical information translates into complex processes like cell growth, differentiation or motility [118,119]. Mechanical energy makes sense in this context as numerous cellular processes involve the activation of stress-sensitive molecules or fluid flow generation. On the other hand, overlooked pathologies caused by low-power sound and low-frequency noise (up to 500 $\mathrm{Hz}$ ) are most likely associated with changes in mechanotransduction cellular signalling [120].

In close mimicry of biological systems, mechanical motion and other chemical responses can be performed by external stimuli. A plethora of micro/nanomotors have been designed in recent years, with varied degree of functionalization, which work under physical force, including ultrasonic acoustic waves [121,122]. Surface acoustic waves (SAWs) that can be generated in miniaturized devices are ideal for lab-on-a-chip applications. A seminal demonstration by $\mathrm{Hu}$ and associates showed how an acoustic needle that vibrates under the action of a piezoelectric transducer was able to trap and rotate small particles around its tip in water. The rotation of trapped particles can be controlled by the acoustic pressure near the tip or by controlling the frequency and power (via voltage) of the transducer [123]. An extension of this concept is the use of SAWs on a piezoelectric substrate (made of lithium niobate) with a pair of interdigital transducers to induce rotation of a small disk ( $5 \mathrm{~mm}$ diameter) immersed in a water drop. Large radial accelerations $\left(172 \mathrm{~m} / \mathrm{s}^{2}\right)$ were obtained and hold potential for microcentrifugation [124].

The autonomous motion of metallic microrods, in the form of levitation, rotation, propulsion, alignment and particle assembly has been induced in water or saline solutions using SAWs at 3.7 MHz [125]. Moreover, results obtained with metallic and polymer 
(polystyrene) materials are substantially different and depend strongly on shape and symmetry (Figure 23). Metal rods induce strong vortices upon alignment and display preferential axial motion, while spherical metal particles induce vortices as well, but scarcely show directional motion. Polymer particles show neither directional motion nor induce vortex formation, irrespective of shape. The fact that polymer rods display no directional motion, but weak axial rotation, when aligned suggest that such trends are influenced by different acoustic field effects. Thus, axial propulsion arises chiefly from the scattering of acoustic waves travelling in the $z$-direction and its rate can be altered either by modifying the amplitude and frequency of the continuous irradiation or by means of pulsed sonication. It would appear that the swimming trajectory of an object is governed by the balance between the orienting force induced by the physical field and the effects that randomize particle orientations, such as rotary Brownian motion and/or a tumbling mechanism like that of flagella in bacteria [126].

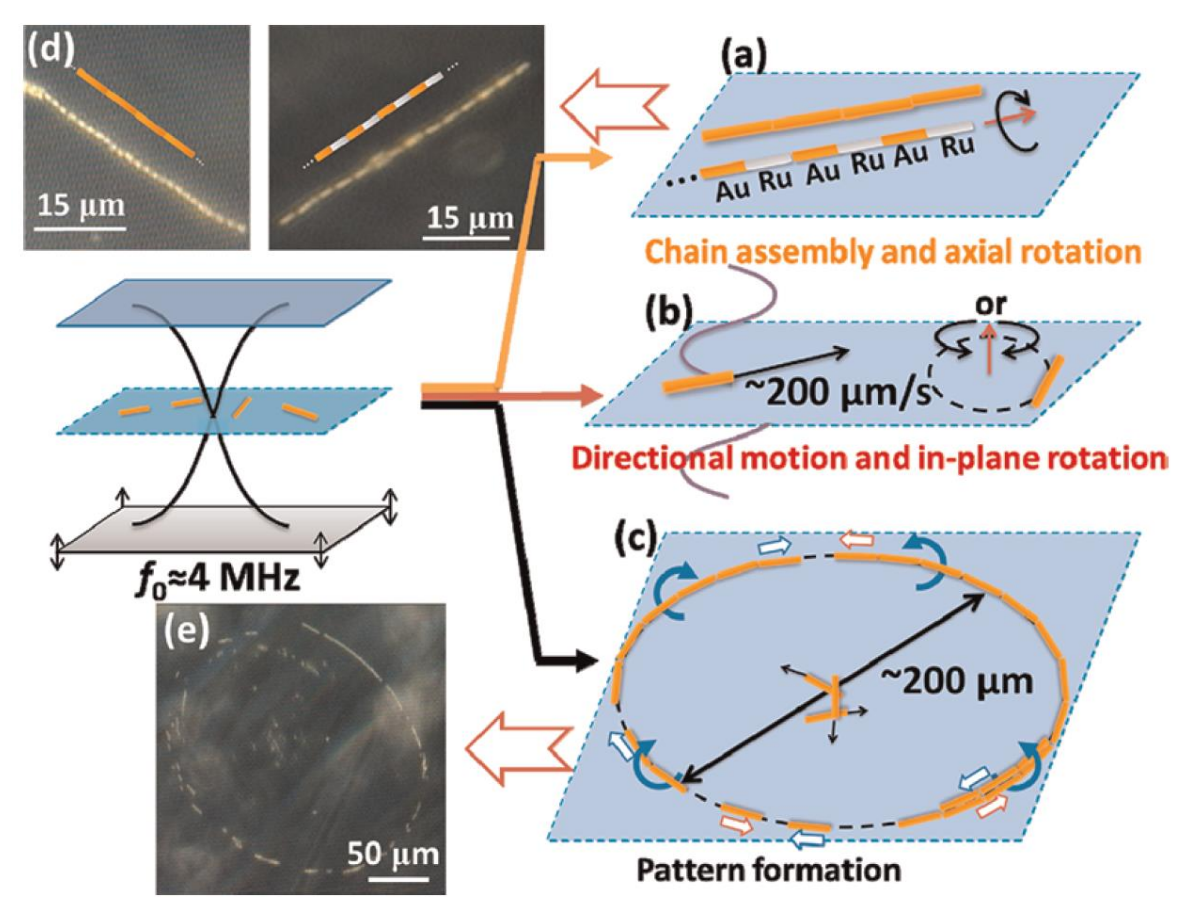

Fig. 23. Schematic illustration of the different types of motion: (a) axial directional motion with chain assembly, (b) in-plane rotation, and (c) axial spinning and pattern formation, of metal microrods in a 3.7-MHz acoustic field. AuRu rods (gold-silvery colour in dark image) showed similar behaviour to of Au rods, except that they moved from the Ru ends (silvery end in the image) forward and aligned heat-to-tail into chains. Images (d) and (e) show chain structures and ring patterns formed by Au and AuRu rods. Reproduced with permission from ref. [125]. Copyright 2012 American Chemical Society. 
Wang et al. have recently displayed the modulating effect of an ultrasonic field on the bubble propulsion of a chemically-fuelled microengine [127]. The latter, fabricated by electrodeposition, consists of a poly(3,4-ethylenedioxythiophene) (PEDOT)/Ni/Pt tubular arrangement $(15 \mu \mathrm{m}$ long, $5 \mu \mathrm{m}$ diameter in its wide side). The catalytic decomposition of hydrogen peroxide fuel on the inner Pt surface releases oxygen microbubbles, which leads to microengine propulsion. The application of ultrasound disrupts bubble evolution, thus hindering and stopping movement. In the absence of sonication, the microengine moves at high speed $(231 \mu \mathrm{m} / \mathrm{s})$ with a characteristic bubble tail. When irradiation is applied (at $10 \mathrm{~V}$ of the US transducer), the microengine almost stops completely within $0.1 \mathrm{~s}$ and only maintains a negligible velocity of $0.6 \mu \mathrm{m} / \mathrm{s}$. When the ultrasonic field is turned off, the original speed is regained once again within $0.1 \mathrm{~s}$. Reproducible on/off cycles are observed and different velocities can be obtained by simply tuning ultrasonic power.

Acoustic propulsion in biological fluids and cells opens the door to biomedical applications, from drug delivery to the selective transport or capture of biological targets (e.g. bacteria) [128], and have perhaps taken their inspiration from the film "Fantastic Voyage". The fuel-free propulsion of a nanomotor by means of acoustic energy can be additionally oriented in the presence of a magnetic field, thus targeting specific cells and tissues. As a proof of concept, a ferromagnetic nickel stripe has been electrochemically grown between diamagnetic $\mathrm{Au}$ and $\mathrm{Ru}$ segments, which then respond to a weak external magnetic field (40-45 $\mathrm{mT})$ used to orient nanowires that move along their long axes, propelled by SAWs $(\sim 3.7 \mathrm{MHz})[129]$. These features enable $\mu \mathrm{m}$-level precision toward living human cervical cancer cells (HeLa cells) cultured in an aqueous phosphate buffer (Figure 24). Moreover, viability tests showed no significant cell degradation in the presence of metallic nanowires after $20 \mathrm{~min}$ of acoustic irradiation (at $10 \mathrm{~V}$ peak values). This experiment demonstrates clever random motion suppression in microengines which were fine-tuned by a second controlling field. Ultrasound-excited motion (at $\sim 4 \mathrm{MHz}$ resonant frequency) of $\mathrm{Au}$ nanorods inside $\mathrm{HeLa}$ cells, which remain viable after ultrasonic exposure, has also been reported and shows the aforementioned directionality patterns of axial propulsion and spinning [130]. 


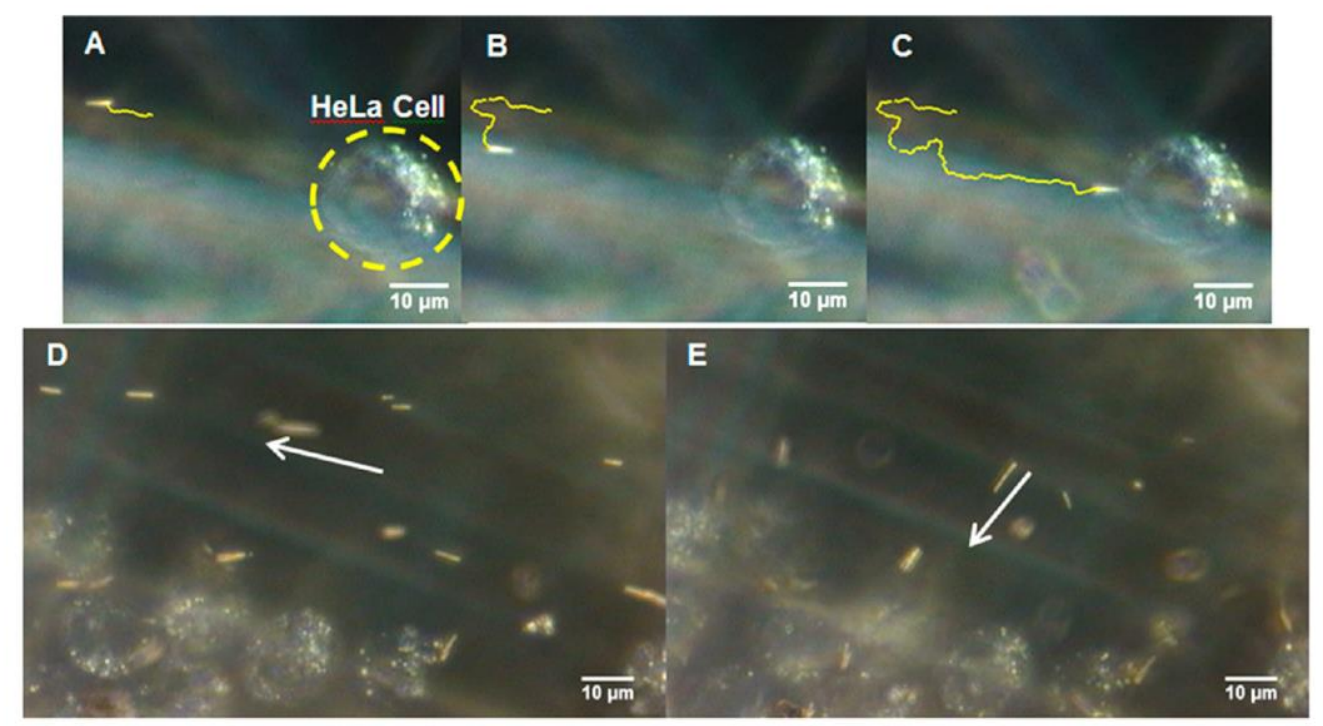

Fig. 24. Acoustically-propelled nanomotor targeted at a HeLa cell (images A-C). D: nanowires are moving parallel to a group of cells, with their direction indicated by the arrow. E: when the external field is turned, nanowires are magnetically steered toward the cells through an approximately $90^{\circ}$ turn. Reproduced with permission from ref. [129]. Copyright 2013 American Chemical Society.

In a different strategy to achieve ultrasound-guided motion, Wang and coworkers prepared microbullets with an inner Au layer that permits conjugation to a monolayer of thiolated cysteamine. The entire functionalization also enables electrostatic attachment to perfluorocarbon (PFC) (either perfluoropentane or perfluorohexane) droplets to be carried out. Under ultrasonic irradiation, the PFC droplets are vaporized, leading to the net motion of microbullets towards lamb kidney tissue. Propulsion can be modulated by adjusting external parameters like acoustic pressure, pulse duration or surfactant concentration [131].

Although not directly related to acoustic nanomotors, one might wish to pay attention to the selective binding control of single molecules in biological media. As an illustrative example, low-power ultrasound radiation, mediated by magnetic particles, can selectively dissociate non-covalent bonds according to their inherent strengths [132]. Consider for instance, as shown in Figure 25, two types of non-covalent bonds to biological receptors: one occurring between a magnetically labelled ligand and receptor 1; the other involving interaction between the ligand and receptor 2, that is assumed to have a weaker binding constant than the former. Low-power sonication will only be able to selectively dissociate the weaker bond. The dissociated magnetic particles will cause a decrease in the magnetic signal due to the randomization of their magnetic dipoles. Subsequent application of a slightly higher-power ultrasound will dissociate the stronger 
bond between the ligand and receptor 1. Different antibodies and DNA duplexes have been mechanically resolved using this approach.

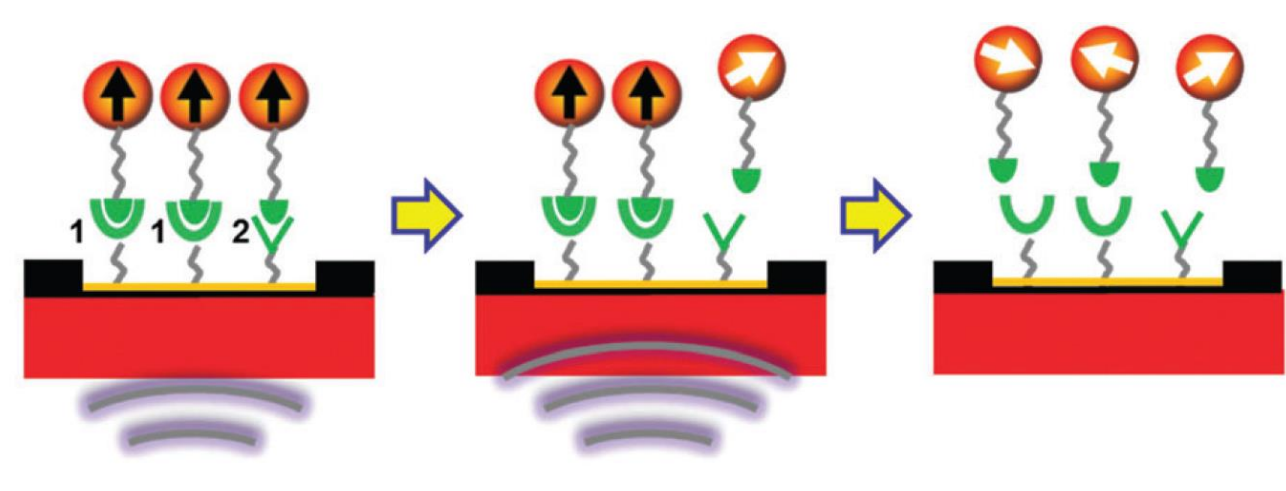

Aligned magnetic particles

Ultrasound radiation

Random magnetic particles

Attenuator

Fig. 25. Schematic illustration of the acoustic radiation force (ARF)-based FIRMS (force-induced remnant magnetization spectroscopy) approach for the selective cleavage of non-covalent bonds. Reproduced with permission from ref. [132]. Copyright 2014 The Royal Society of Chemistry.

\section{Cleaning, Erosion and Streaming: New Developments}

Cleaning, erosion, emulsification and streaming are paradigmatic effects of the mechanical action of high-amplitude waves and cavitating bubbles. This penultimate section does not intend to re-emphasize these aspects, as they are well established and lie at the core of sonochemistry. Rather, we shall provide glimpses of a few recent applications which harness these mechanical mechanisms.

Fluid recirculation enhancing acoustic streaming [26], can be used in the sonoprocessing of solid particles and, most notably, make use of low-cost commercially available devices which operate at low frequencies. The so-called Ca-looping process is a viable technology with which to perform postcombustion $\mathrm{CO}_{2}$ capture with high efficiency. In short, it involves the separation of $\mathrm{CO}_{2}$ via the carbonation of $\mathrm{CaO}$ in a fluidized bed at high temperature, followed by limestone calcination to regenerate the sorbent. Capture is increased under low frequency acoustic vibration (ca. $100 \mathrm{~Hz}$ ) and intensities of around $140 \mathrm{~dB}[133,134]$. The operational setup is shown in Figure 26. Fine particles (with diameters smaller than $100 \mu \mathrm{m}$ ) are entrained in the oscillating gas flow induced by the low-frequency field, which leads to the strong agitation of the bed and 
enhances gas-solid contact. Moreover, acoustic streaming (i.e. intense convection of gas flow) is generated on the surface of larger particles (otherwise unmovable by the acoustic wave) and promotes both heat and mass transfer at the gas-solid interface. These mechanisms, used combined or separately, increase carbonation/decarbonation rates, but depend heavily on average particle size and sound parameters.

It should be noted that audible sound (generated by cheap loudspeakers) can be used for related separation techniques, even at large scales (pilot plant). A reduction in particle emissions from coal combustion fumes has been achieved using an array of stepped-plate piezoelectric transducers, which produce a homogeneous distribution of high-intensity waves (145-165 dB), located along the wall of the chamber at that hightemperature environment [135].

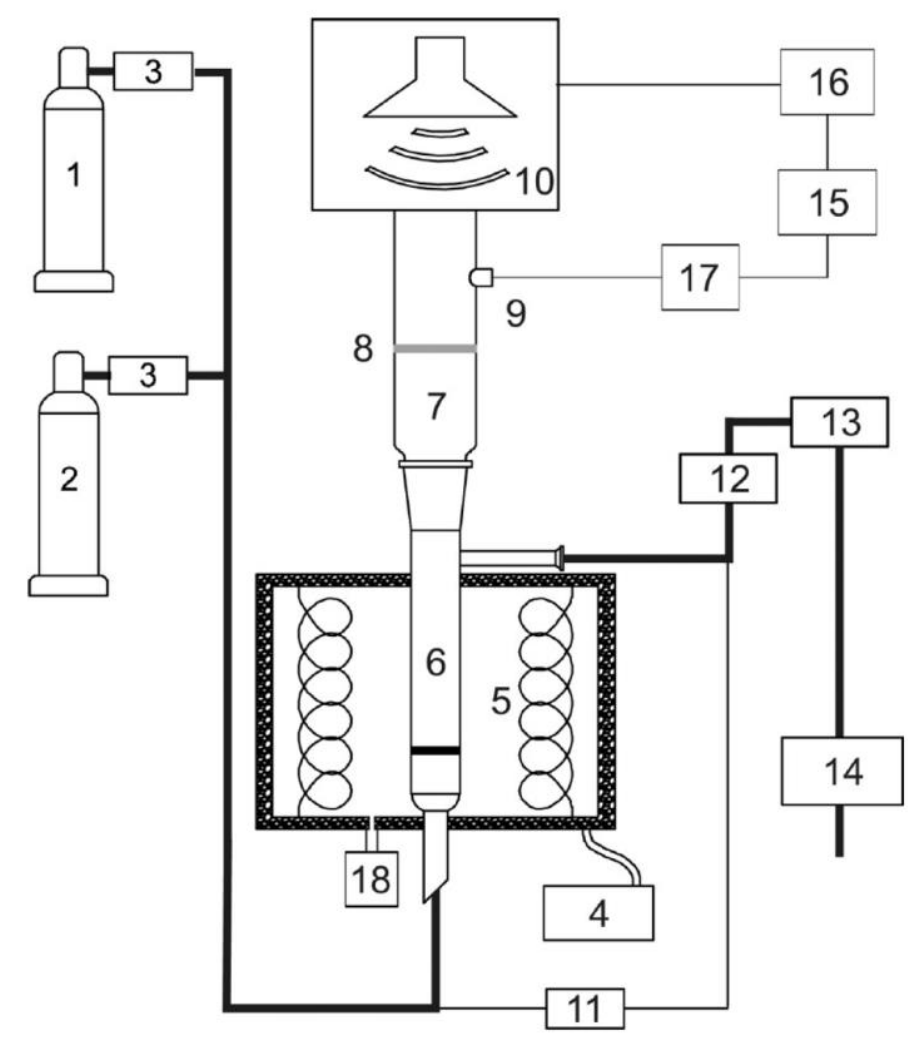

Fig. 26. Diagram showing Ca-looping sonoprocessing. 1: Compressed gas used for carbonation ( $\left.15 \% \mathrm{CO}_{2} / 85 \% \mathrm{~N}_{2} \mathrm{v} / \mathrm{v}\right) ; 2$ : compressed gas used for calcinations (dry air); 3 : mass flow controllers; 4: temperature controller; 5: furnace; 6: quartz reactor; 7: sound waveguide; 8: elastic membrane; 9: microphone; 10: loudspeaker; 11: differential pressure transducer; 12: particulate filter; 13: mass flow meter; 14: gas analyzer; 15: signal amplifier; 16: signal generator; 17: oscilloscope; 18: air cooling system. Reproduced with permission from ref. [133]. Copyright 2013 American Chemical Society. 
Cleaning and erosion can be visualized at small-scales using miniaturized devices and provide fine details of the mechanical action. Material removal experiments have been conducted using the setup shown in Figure 21 (top) for the elimination of biofilms [110]. The cavitation cell does not enable an optical visualization of the cavitating bubbles due to the opaque character of materials deposited on the glass slide. However, some observations, which are also dependent on the pit arrangement and intensity (Figure 27), were made. For deposited gold, a small opening in the Au layer (right on top) is seen (where the pits are located) when low power irradiation is applied $(0.182 \mathrm{~W})$. At constant power, the size of the removed layer increases slowly. The maximum area removed for one pit is ca. $0.03 \mathrm{~mm}^{2}$. This increases proportionally upon increasing the power for two, three or four pit geometries (linear, triangular or rectangular shapes, respectively, are seen). While this kind of surface removal is observed for both $\mathrm{Au}$ and $\mathrm{Pt}$, deposited $\mathrm{Cr}$ and $\mathrm{Ti}$ (expected to have harder passivating coatings) do not undergo removal under the above conditions and the bubbles are able to detach small pieces only occasionally. When the ultrasound is switched off, the pits are filled with water and, if a bubble is still inside a pit, further bubble nucleation can no longer be attained. When ultrasound is turned on, no cavitation is observed from the micropits. As mentioned in Sect. 4, streaming does not account for surface removal and the cleaning effect is locally restricted to the pits and how close the nucleated bubbles are from the surface to be removed.

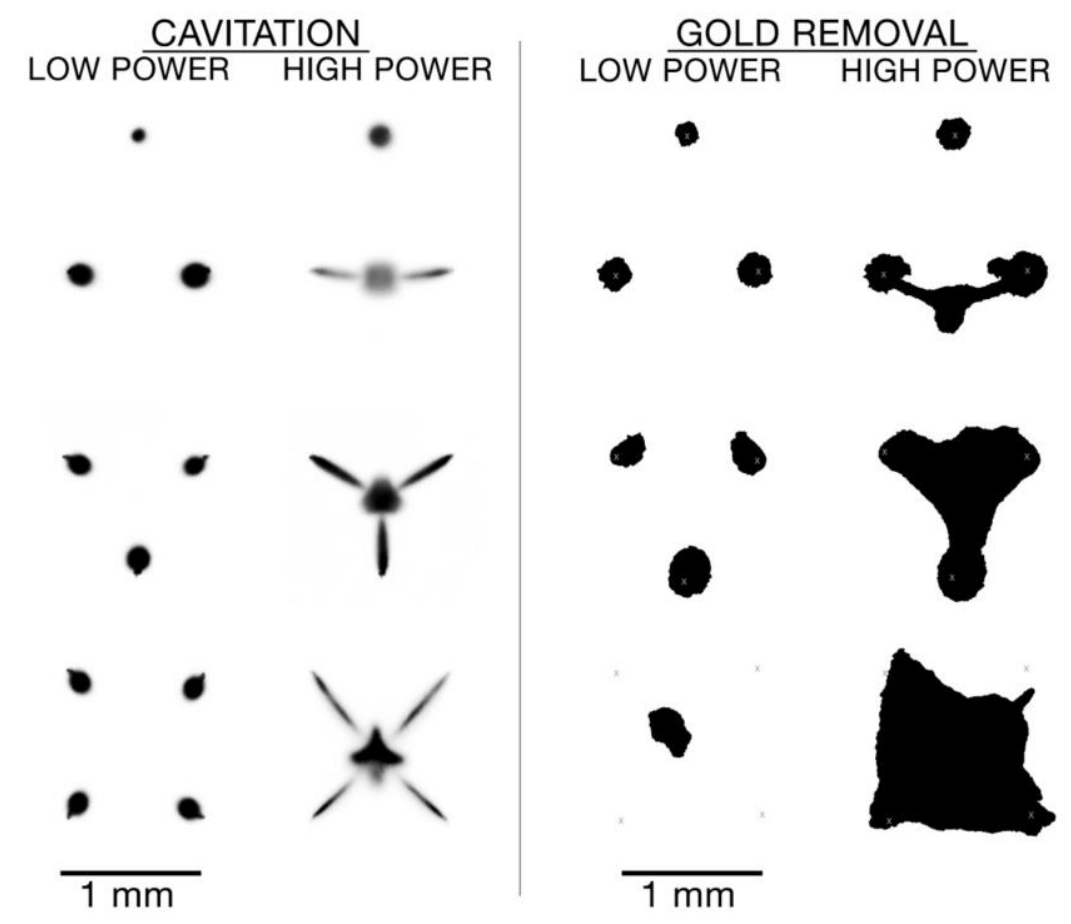

Fig. 27. From top to bottom (and left to right): bubble generation from 1 to 4 pit arrangements at low and high power. Left: bright field illumination (inverted colour) showing bubble patterns at slow speed imaging. Right: Shapes of cleaned areas in gold 
(black) caused by bubble cavitation. Reproduced with permission from ref. [110]. Copyright 2012 AIP Publishing.

The influence of radical production by the cavitation bubbles was also investigated by exposing all the metal surfaces to hydrogen peroxide. No Au reaction was observed in the first $5 \mathrm{~min}$, however some small regions showed tiny amounts of detachment (ca. 1 $\mathrm{mm}^{2}$ ) after that time. Some chemical activity was detected in Pt in the form of bubbling and gas formation, most likely $\mathrm{H}_{2} / \mathrm{O}_{2}$ generation, which led to the removal of the entire layer in a few seconds. On the other hand, $\mathrm{Cr}$ and $\mathrm{Ti}$ did not undergo reaction after 10 min of irradiation. The latter allows to conjecture that any contribution from radical species (such as $\mathrm{H} \cdot$ or $\mathrm{HO} \cdot$ ) to the removal rates will be minimal under the conditions employed to produce cavitating bubbles, which nucleate from the pits [110].

Surface damage can be caused by bubble cavitation and shock waves on noncoinage metals. Silicon is an oft-studied case where surface erosion has been studied in various crystallographic orientations; namely (100), (110) and (111) surfaces at $191 \mathrm{kHz}$ [136]. The most significant damage caused by cavitation was observed on the (100) face, with an eroded area which was ca. 2.5 times larger than in other crystallographic orientations after $180 \mathrm{~min}$ of irradiation. Erosion pits increased at a constant rate for (110) and (111), although this effect stopped in (100) after $120 \mathrm{~min}$.

More complex transformations have been observed for crystalline silicon under acoustic cavitation in water sparged with Ar at temperatures of between 10 and $20^{\circ} \mathrm{C}$ [137]. Spectroscopic investigation reveals that Ar, which is bubbled continuously through the liquid phase, is ultrasonically excited via mechanoluminescence, i.e. light emission produced by mechanical action on the Si surface. This phenomenon also triggers physicochemical transformations at the solid-liquid interface (Figure 28), thus causing stress and defects as well as an increase in roughness and wettability. Interestingly, progressive amorphization of the crystalline was also observed. The latter can be attributed to a loss of lattice stability during the high-energy environment created by the collapsing bubbles in compression/decompression cycles. This shock-wave-caused mechanical effect should also be considered as the local pressures generated by such waves are similar to those employed in high-pressure transformations of crystalline silicon. 

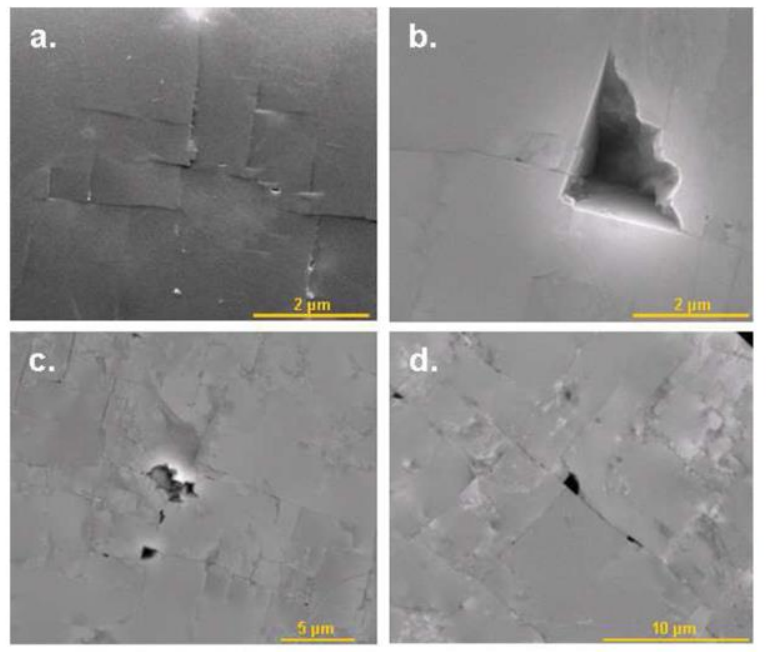
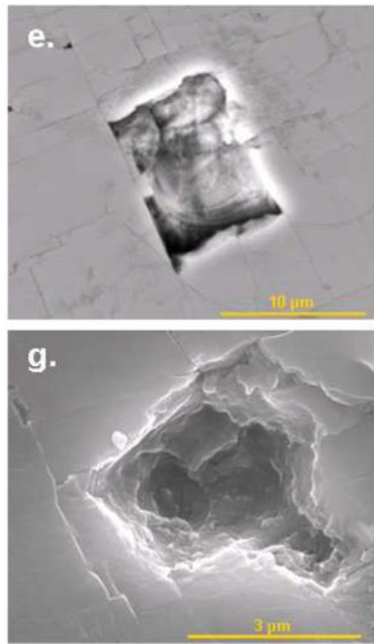
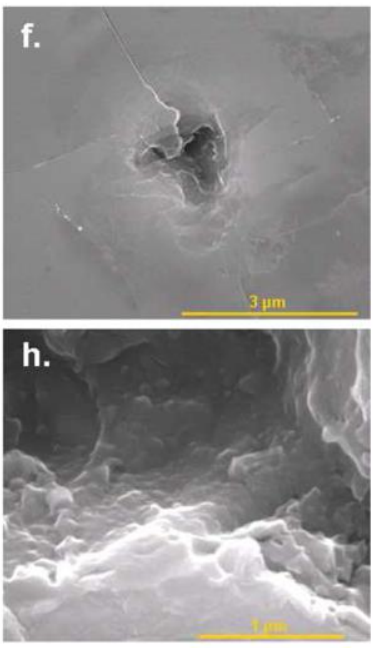

Fig. 28. SEM images of crystalline silicon showing the propagation of cracks and defects after sonication for (a) $5 \mathrm{~h}$, both (b) and (c) $7 \mathrm{~h}$, (d) $9 \mathrm{~h}$, (e) $12 \mathrm{~h}$, and (f)-(h) $15 \mathrm{~h}$. Acoustic intensity is $32 \mathrm{~W} / \mathrm{cm}^{2}$, Ar bubbling at $20{ }^{\circ} \mathrm{C}$ in water $(250 \mathrm{~mL})$. Reproduced with permission from ref. [137]. Copyright 2012 American Chemical Society.

\section{Concluding Remarks: Open Questions}

It should now be unnecessary to underline the mechanical connection between sonochemistry and other subfields of mechanochemistry. The former clearly possesses a series of inherent characteristics by virtue of various forces generated in a liquid under the action of pressure waves. Both chemical and physical activation, especially when cavitation is present, are able to drive numerous transformations and often provide a useful mechanistic rationale.

As a leading sonochemist [9], who was paraphrasing Churchill, once said; sonochemists and mechanochemists are one people separated by a common phenomenon. This phenomenon is obviously the conversion of mechanical energy into chemistry, which includes a vast territory of applications worthy of exploration. The present survey simply summarizes a few fundamentals plus some scenarios of current and active interest. The interplay between sonochemistry and its mechanical relationships invariably leaves open questions and new avenues to investigate. To mention a few:

a) Further developments in theoretical modelling, particularly via high-level quantum-mechanical calculations, are required and will boost our understanding of the forces involved. Cavitational modelling is, in any case, a challenge because of its nonlinear effects. 
b) How can selectivity be controlled in macromolecular or supramolecular arrangements?

c) What kind of physical effects are involved in the acoustically-promoted formation of metastable crystals, co-crystals and nanostructures?

d) How do cavitation and other physical effects influence or alter phenomena such as mechanoluminescence or chemiluminescence?

e) How does reactor design affect efficiency and reproducibility?

f) Should energy efficiency also be analyzed in terms of batch versus continuous processes?

g) How different are acoustic fields from other forms of flowing energy? (e.g. hydrodynamic cavitation)

h) What kind of safety concerns should be addressed? (in particular mechanical input in single cells and biofluids)

i) How can we assess sonomechanical mechanisms? Are there suitable molecular probes?

j) Can we probe cell mechanics with ultrasonics via non-invasive and innocuous techniques?

Ultrasonication tells us that liquid flows may offer rich science, merging physics and chemistry. We are often fascinated by liquid patterns observed in common and cheap cleaning baths or bubble clouds around an ultrasonic horn. Many years ago, Leonardo da Vinci, the prototypical Renaissance genius, was the man who loved fluids [138]. His drawings reflect passion and curiosity and show swirling, curving, revolving and wavy patterns with precision. He recognized that flow was an essential ingredient of life. Had Leonardo known more about the action of sound in liquids, he would have certainly conveyed science and beauty to mesmerize all future generations forever.

\section{Acknowledgments}

Financial support from the following agencies is gratefully acknowledged: University of Turin (fondi ricerca locale 2013) and the Junta de Extremadura-FEDER (Ayuda a Grupos Consolidados, Grant No. GR10049). The authors are also deeply indebted to Dr. David Fernández-Rivas (University of Twente, The Netherlands, and BubClean) for his stimulating feedback and permission to reproduce Figure 4.

\section{References}

1. Gooberman GL (1990) Sound. In: Gwinn RP, Norton PB, Goetz PW (eds) The new encyclopaedia britannica vol. 27, Encyclopaedia Britannica Inc, Chicago, pp 629-631 (ultrasonics); entire chapter on sound: pp 604-632 
2. Rossing TD (2007) Introduction to acoustics. In: Rossing TD (ed) Springer handbook of acoustics, Springer, New York, pp 1-6

3. Cravotto G, Cintas P (2011) Introduction to sonochemistry: a historical and conceptual overview. In: Chen D, Sharma, SK, Mudhoo A (eds) Handbook on applications of ultrasound and sonochemistry, CRC Press-Taylor \& Francis group, Boca Raton, FL, Ch 2, pp 23-40

4. Mason TJ, Lorimer JP (2002) Applied sonochemistry. The uses of power ultrasound in chemistry and processing, Wiley-VCH, Weinheim

5. Cravotto G, Cintas P (2006) Power ultrasound in organic synthesis: moving cavitational chemistry from academia to innovative and large-scale applications. Chem Soc Rev 35:180-196

6. Caruso MM, Davis DA, Shen Q, Odom SA, Sottos NR, White SR, Moore JS (2009) Mechanically-induced chemical changes in polymeric materials. Chem Rev 109:5755-5798

7. Cravotto G, Cintas P (2012) Harnessing mechanochemical effects with ultrasoundinduced reactions. Chem Sci 3:295-307

8. Cravotto G, Calcio Gaudino E, Cintas P (2013) On the mechanochemical activation by ultrasound. Chem Soc Rev 42:7521-7534

9. Suslick KS (2014) Mechanochemistry and sonochemistry: concluding remarks. Faraday Discuss 170: (doi: 10.1039/c4fd00148f)

10. May PA, Moore JS (2013) Polymer mechanochemistry: techniques to generate molecular force via elongational flows. Chem Soc Rev 42:7497-7506

11. Wiggins KM, Brantley JN, Bielawski CW (2013) Methods for activating and characterizing mechanically responsive polymers. Chem Soc Rev 42:7130-7147

12. Brantley JN, Wiggins KM, Bielawski CW (2013) Polymer mechanochemistry: the design and study of mechanophores. Polym Int 62:2-12

13. Bang JH, Suslick KS (2010) Applications of ultrasound to the synthesis of nanostructured materials. Adv Mater 22:1039-1059.

14. Ariga K, Mori T, Hill JP (2012) Mechanical control of nanomaterials and nanosystems. Adv Mater 24:158-176

15. Xu H, Zeiger BW, Suslick KS (2013) Sonochemical synthesis of nanomaterials. Chem Soc Rev 42:2555-2567

16. Sander JRG, Zeiger BW, Suslick KS (2014) Sonocrystallization and sonofragmentation. Ultrason Sonochem 21:1908-1915

17. Huang Z, Boulatov R (2011) Chemomechanics: chemical kinetics for multiscale phenomena. Chem Soc Rev 40:2359-2384.

18. Ribas-Arino J, Marx D (2012) Covalent mechanochemistry: theoretical concepts and computational tools with applications to molecular nanomechanics. Chem Rev 112:5412-5487

19. Kildishev AV, Boltasseva A, Shalaev VM (2013) Planar photonics with metasurfaces. Science 339:1232009 (doi: 10.1126/science.1232009) 
20. Brunet T, Leng J, Mondain-Monval O (2013) Soft acoustic metamaterials. Science 342:323-324

21. Maldovan M (2013) Sound and heat revolutions in phononics. Nature 503:209-217

22. Gustafsson MV, Aref T, Kockum AF, Ekström MK, Johansson G, Delsing P (2014) Propagating phonons coupled to an artificial atom. Science 346:207-211

23. Humphrey VF (2007) Ultrasound and matter-physical interactions. Prog Biophys Mol Biol 93:195-211

24. Lepoint T, Lepoint-Mullie F (1998) Theoretical bases. In: Luche JL (ed) Synthetic organic sonochemistry, Plenum Press, New York, Ch 1, pp 1-49

25. Nyborg WL (1998) Acoustic streaming. In: Hamilton MF, Blackstock DT (eds) Nonlinear acoustics, Academic Press, San Diego, pp 207-228

26. Valverde JM (2013) Acoustic streaming in gas-fluidized beds of small particle. Soft Matter 9:8792-8814

27. Mason TJ, Peters D (2002) Practical sonochemistry. Power ultrasound uses and applications, 2nd ed, Woodhead Publishing, Oxford, pp 1-46

28. Fernández Rivas D (2012) Taming acoustic cavitation, $\mathrm{PhD}$ thesis, University of Twente, The Netherlands, Ch 2, p 10 (doi: 10.3990/1.9789036534192)

29. Mason TJ, Cobley AJ, Graves JE, Morgan D (2011) New evidence for the inverse dependence of mechanical and chemical effects on the frequency of ultrasound. Ultrason Sonochem 18:226-230

30. Portenlänger G, Heusinger H (1997) The influence of frequency on the mechanical and radical effects for the ultrasonic degradation of dextranes. Ultrason Sonochem 4:127-130

31. Tran KVB, Kimura T, Kondo T, Koda S (2014) Quantification of frequency dependence of mechanical effects induced by ultrasound. Ultrason Sonochem 21:716721

32. Tudela I, Sáez V, Esclapez MD, Díez-García MI, Bonete P, González-García J (2014) Simulation of the spatial distribution of the acoustic pressure in sonochemical reactors with numerical methods: a review. Ultrason Sonochem 21:909-919

33. Son Y, Lim M, Ashokkumar M, Khim J (2011) Geometric optimization of sonoreactors for the enhancement of sonochemical activity. J Phys Chem C 115:40964103

34. Merouani S, Ferkous H, Hamdaoui O, Rezgui Y, Guemini M (2015) A method for predicting the number of active bubbles in sonochemical reactors. Ultrason Sonochem 22:51-58

35. Alvarez M, Friend JR, Yeo LY (2008) Surface vibration induced spatial ordering of periodic polymeric patterns on a substrate. Langmuir 24:10629-10632.

36. Friend JR, Yeo LY, Arifin DR, Mechler A (2008) Evaporative self-assembly assisted synthesis of polymeric nanoparticles by surface acoustic wave atomization. Nanotechnology 19:145301

37. Shilton R, Tan MK, Yeo LY, Friend JR (2008) Particle concentration and mixing in microdrops driven by focused surface acoustic waves. J Appl Phys 104:014910 
38. Wu C, Zaitsev VY, Zhigilei LV (2013) Acoustic enhancement of surface diffusion. J Phys Chem C 117:9252-9258

39. Kelling S, Mitrelias T, Matsumoto Y, Ostanin VP, King DA (1997) Acoustic wave enhancement of the catalytic oxidation of carbon monoxide over $\operatorname{Pt}\{110\}$. J Chem Phys 107:5609-5612

40. Inoue Y (2007) Effects of acoustic waves-induced dynamic lattice distortion on catalytic and adsorptive properties of metal, alloy and metal oxide surfaces. Surf Sci Rep 62:305-336

41.Zinovev AV, Veryovkin LV, Moore JF, Pellin MJ (2007) Laser-driven acoustic desorption of organic molecules from back-irradiated solid foils. Anal Chem 79:82328241

42. Dow AM, Wittrig AR, Kenttämaa HI (2012) Laser-induced acoustic desorption (LIAD) mass spectrometry. Eur J Mass Spectrom 18:77-92

43. Lipeles R, Kivelson D (1980) Experimental studies of acoustically induced birefringence. J Chem Phys 72:6199-6208

44. Nomura H, Matsuoka T, Koda S (2004) Ultrasonically induced birefringence in polymer solution. Pure Appl Chem 76:97-104

45. Khunsin W, Amann A, Kocher-Oberlehner G, Romanov SG, Pullteap S, Seat HC, O'Reilly EP, Zentel R, Torres CMS (2012) Noise-assisted crystallization of opal films. Adv Funct Mater 22: 1812-1821

46. Avetissov I, Sadovskiy A, Belov S, Khomyakov A, Rekunov K, Kostikov V, Sukhanova E (2013). Thermodynamic features of axial vibrational control technique for crystal growth from the melt. CrystEngComm 15:2213-2219

47. Ende DJA, Anderson SR, Salan JS (2014) Development and scale-up of cocrystals using resonant acoustic mixing. Org Process Res Dev 18:331-341

48. Liu C, Wu P, Wang L (2013) Particle climbing along a vibrating tube: a vibrating tube that acts as a pump for lifting granular materials from a silo. Soft Matter 9:47624766

49. Cravotto G, Cintas P (2009) Molecular self-assembly and patterning induced by sound waves. The case of gelation. Chem Soc Rev 38:2684-2697

50. Yu X, Chen L, Zhang M, Yi T (2014) Low-molecular-mass gels responding to ultrasound and mechanical stress: towards self-healing materials. Chem Soc Rev 43:5346-5371.

51. Ye E, Chee PL, Prasad A, Fang X, Owh C, Yeo VJJ, Loh XJ (2014) Supramolecular soft biomaterials for biomedical applications. Materials Today 17:194-202

52. Naota T, Koori H (2005) Molecules that assemble by sound: an application to the instant gelation of stable organic fluids. J Am Chem Soc 127:9324-9325

53. Bardelang D, Zaman MB, Moudrakovski IL, Pawsey S, Margeson JC, Wang D, Wu X, Ripmeester JA, Ratcliffe CI, Yu K (2008) Interfacing supramolecular gels and quantum dots with ultrasound: smart photoluminescent dipeptide gels. Adv Mater 20:4517-4520 
54. Anderson KM, Day GM, Paterson MJ, Byrne P, Clarke N, Steed JW (2008) Structure calculations of an elastic hydrogel from sonication of rigid small molecule components. Angew Chem Int Ed 47:1058-1062

55. Ke D, Zhan C, Li ADQ, Yao J (2011) Morphological transformation between nanofibers and vesicles in a controllable bipyridine-tripeptide self-assembly. Angew Chem Int Ed 50:3715-3719

56.Zhang M, Jiang M, Meng L, Liu K, Mao Y, Yi T (2013) Fabrication of multiplicate nanostructures via manipulation of the self-assembly between an adamantine based gelator and cyclodextrin. Soft Matter 9:9449-9454

57. Datskos P, Chen J, Sharma J (2014) Synthesis of very small diameter silica nanofibers using sound waves. Chem Commun 50:7277-7279

58. Koenig M, Torres T, Barone V, Brancato G, Guldi DM, Bottari G (2014) Ultrasoundinduced transformation of fluorescent organic nanoparticles from a molecular rotor into rhomboidal nanocrystals with enhanced emission. Chem Commun 50:1295512958

59. Sun H, Zhang Y, Yan W, Chen W, Lan Q, Liu S, Jiang L, Chi Z, Chen X, Xu J (2014) A novel ultrasound-sensitive mechanofluorochromic AIE-compound with remarkable blue-shifting and enhanced emission. J Mater Chem C 2:5812-5817

60. Kostarelos K, Novoselov KS (2014) Exploring the interface of graphene and biology. Science 344:261-263

61. Nicolosi V, Chhowalla M, Kanatzidis MG, Strano MS, Coleman JN (2013) Liquid exfoliation of layered materials. Science 340:1226419 (doi: 10.1126/science.1226419)

62. Cravotto G, Cintas P (2010) Sonication-assisted fabrication and post-synthetic modification of graphene-like materials. Chem Eur J 16:5246-5259

63. Paton KR, Varrla E, Backes C, Smith RJ, Khan U, O’Neill A, Boland C, Lotya M, Istrate OM, King P, Higgins T, Barwich S, May P, Puczharski P, Ahmed I, Moebius M, Pettersson H, Long E, Coelho J, O'Brien SE, McGuire EK, Sanchez BM, Duesberg GS, McEvoy N, Pennycook TJ, Downing C, Crossley A, Nicolosi V, Coleman JN (2014) Scalable production of large quantities of defect-free few-layer graphene by shear exfoliation in liquids. Nat Mater 13:624-630

64. Buzaglo M, Shtein M, Kober S, Lovrincic R, Vilan A, Regev O (2013) Critical parameters in exfoliating graphite into graphene. Phys Chem Chem Phys 15:44284435

65. Sesis A, Hodnett M, Memoli G, Wain AJ, Jurewicz I, Dalton AB, Casey JD (2013) Influence of acoustic cavitation on the controlled ultrasonic dispersions of carbon nanotubes. J Phys Chem B 117:15141-15150

66. Bracamonte MV, Lacconi GI, Urreta SE, Foa Torres LEF (2014) On the nature of defects in liquid-phase exfoliated graphene. J Phys Chem C 118:15455-15459

67. Janowska I, Chizari K, Ersen O, Zafeiratos S, Soubane D, Da Costa V, Speisser V, Boeglin C, Houllé M, Bégin D, Plee D, Ledoux MJ, Pham-Huu C (2010) Microwave synthesis of large few-layer graphene sheets in aqueous solution of ammonia. Nano Res 3:126-137 
68. Zhu Y, Murali S, Stoller MD, Ganesh KJ, Cai W, Ferreira PJ, Pirkle A, Wallace RM, Cychosz KA, Thommes M, Su D, Stach EA, Ruoff RS (2011) Carbon-based supercapacitors produced by activation of graphene. Science 332:1537-1541

69. Cravotto G, Garella D, Calcio Gaudino E, Turci F, Bertarione S, Agostini G, Cesano F, Scarano D (2011) Rapid purification/oxidation of multi-walled carbon nanotubes under $300 \mathrm{kHz}$-ultrasound and microwave irradiation. New J Chem 35:915-919

70.Zheng J, Liu HT, Wu B, Di CA, Guo YL, Wu T, Yu G, Liu YQ, Zhu DB (2012). Production of graphite chloride and bromide using microwave sparks. Sci Rep 2:662 (doi: 10.1038/srep00662)

71. Economopoulos SP, Rotas G, Miyata Y, Shinohara H, Tagmatarchis N (2010) Exfoliation and chemical modification using microwave irradiation affording highly functionalized graphene. ACS Nano 4:7499-7507

72. Kissel P, Murray DJ, Wulftange WJ, Catalano VJ, King BT (2014) A nanoporous two-dimensional polymer by single-crystal-to-single-crystal photopolymerization. Nat Chem 6:774-778

73. Kory MJ, Wörle M, Weber T, Payamyar P, van de Poll SW, Dshemuchadse J, Trapp N, Schlüter AD (2014) Gram-scale synthesis of two-dimensional polymer crystals and their structure analysis by X-ray diffraction. Nat Chem 6:779-784

74. Encina MV, Lissi E, Sarasúa M, Garagallo L, Radic D (1980) Ultrasonic degradation of polyvinylpyrrolidone: effect of peroxide linkages. J Polym Sci Polym Lett Ed 18:757-760.

75. Berkowski KL, Potisek SL, Hickenboth CR, Moore JS (2005) Ultrasound-induced site-specific cleavage of azo-functionalized poly(ethylene glycol). Macromolecules 38:8975-8978

76. Hickenboth CR, Moore JS, White SR, Sottos NR, Baudry J, Wilson SR (2007) Biasing reaction pathways with mechanical force. Nature 446:423-427

77. Luty T, Ordon P, Eckhardt CJ (2002) A model for mechanochemical transformations: applications to molecular hardness, instabilities, and shock initiation of reaction. J Chem Phys 117:1775-1785

78. Tian Y, Boulatov R (2013) Comparison of the predictive performance of the BellEvans, Taylor-expansion and statistical-mechanics models of mechanochemistry. Chem Commun 49:4187-4189

79. Nguyen TQ, Liang QZ, Kausch HH (1997) Kinetics of ultrasonic and transient elongational flow degradation: a comparative study. Polymer 38:3783-3793

80. Chen Y, Spiering AJH, Karthikeyan S, Peters GWM, Meijer EW, Sijbesma RP (2012) Mechanically induced chemiluminescence from polymers incorporating a 1,2dioxetane unit in the main chain. Nat Chem 4:559-562

81. Diesendruck CE, Peterson GI, Kulik HJ, Kaitz JA, Mar BD, May PA, White SR, Martinez TJ, Boydston AJ, Moore JS (2014) Mechanically triggered heterolytic unzipping of a low-ceiling-temperature polymer. Nat Chem 6:623-628

82. Larsen MB, Boydston AJ (2014) Successive mechanochemical activation and small molecule release in an elastomeric material. J Am Chem Soc 136:1276-1279 
83. Gossweiler GR, Hewage GB, Soriano G, Wang Q, Welshofer GW, Zhao X, Craig SL (2014) Mechanochemical activation of covalent bonds in polymers with full and repeatable macroscopic shape recovery. ACS Macro Lett 3:216-219

84. Diesendruck CE, Zhu L, Moore JS (2014) Alkyne mechanochemistry: putative activation by transoidal bending. Chem Commun 50:13235-13238

85. McNutt M (2014) Editorial expression of concern. Science 344:1460

86. Halford B (2014) Texas student falsified data. Chem Eng News December 15 issue, p 9

87. Li J, Shiraki T, Hu B, Wright RAE, Zhao B, Moore JS (2014) Mechanophore activation at heterointerfaces. J Am Chem Soc 136:15925-15928

88. Balkenende DWR, Coulibaly S, Balog S, Simon YC, Fiore GL, Weder C (2014) Mechanochemistry with metallosupramolecular polymers. J Am Chem Soc 136:10493-10498

89. Tsuda A, Nagamine Y, Watanabe R, Nagatani Y, Ishii N, Aida, T (2010) Spectroscopic visualization of sound-induced liquid vibrations using a supramolecular nanofibre. Nat Chem 2:977-983

90. Hotta Y, Suiko S, Motoyanagi J, Onishi H, Ihozaki T, Arakawa R, Tsuda A (2014) A physical operation of hydrodynamic orientation of an azobenzene supramolecular assembly with light and sound. Chem Commun 50:5615-5618

91. O'Brien Jr WD (2007) Ultrasound-biophysics mechanisms. Prog Biophys Mol Biol 93:212-255

92. Kennedy JE (2005) High-intensity focused ultrasound in the treatment of solid tumours. Nat Rev Cancer 5:321-327

93. Yu T, Zhang Y, He H, Zhou S, Liu Y, Huang P (2011) Anticancer potency of cytotoxic drugs after exposure to high-intensity focused ultrasound in the presence of microbubbles and hematoporphyrin. Mol Pharmaceutics 8:1408-1415

94. Wang LV, Hu S (2012) Photoacoustic tomography: in vivo imaging from organogelles to organs. Science 335:1458-1462

95. Guo C, Jin Y, Dai Z (2014) Multifunctional ultrasound contrast agents for imaging guided photothermal therapy. Bioconjugate Chem 25:840-854

96. Alvarez-Lorenzo C, Concheiro A (2014) Smart drug delivery systems: from fundamentals to the clinic. Chem Commun 50:7743-7765

97. Tong $\mathrm{R}, \mathrm{Lu} \mathrm{X}$, Xia H (2014) A facile mechanophore functionalization of an amphiphilic block copolymer towards remote ultrasound and redox dual stimulus responsiveness. Chem Commun 50:3575-3578

98. Frenkel V (2008) Ultrasound mediated delivery of drugs and genes to solid tumors. Adv Drug Deliv Rev 60:1193-1208

99. Kang M, Huang G, Leal C (2014) Role of lipid polymorphism in acoustically sensitive liposomes. Soft Matter 10:8846-8854

100. Santo KP, Berkowitz ML (2014) Shock wave induced collapse of arrays of nanobubbles located next to a lipid membrane: coarse-grained computer simulations. J Phys Chem B (doi: 10.1021/jp505720d) 
101. Podaru G, Ogden S, Baxter A, Shrestha T, Ren S, Thapa P, Dani RK, Wang H, Basel MT, Prakash P, Bossmann SH, Chikan V (2014) Pulsed magnetic field induced fast drug release from magneto liposomes via ultrasound generation. J Phys Chem B 118:11715-11722

102. Toublan FJJ, Boppart S, Suslick KS (2006) Tumor targeting by surface-modified protein microspheres. J Am Chem Soc 128:3472-3473

103. Baram-Pinto D, Shukla S, Richman M, Gedanken A, Rahimipour S, Sarid R (2012) Surface-modified protein nanospheres as potential antiviral agents. Chem Commun 48:8359-8361

104. Skirtenko N, Tzanov T, Gedanken A, Rahimipour S (2010) One-step preparation of multifunctional chitosan microspheres by a simple sonochemical method. Chem Eur J 16:562-567

105. Erriu M, Blus C, Szmukler-Moncler S, Buogo S, Levi R, Barbato G, Madonnaripa D, Denotti G, Piras V, Orrù G (2014) Microbial biofilm modulation by ultrasound: current concepts and controversies. Ultrason Sonochem 21:15-22

106. Ensing GT, Roeder BL, Nelson JL, van Horn JR, van der Mei HC, Busscher HJ, Pitt WG (2005) Effect of pulsed ultrasound in combination with gentamicin on bacterial viability in biofilms on bone cements in vivo. J Appl Microbiol 99:443-448

107. Bigelow TA, Northagen T, Hill TM, Sailer FC (2009) The destruction of Escherichia coli biofilms using high-intensity focused ultrasound. Ultrasound Med Biol 35:1026-1031

108. Miller DL (1976) Instrument for microscopical observation of the biophysical effects of ultrasound. J Acoust Soc Am 60:1203-1212

109. Iida Y, Tuziuti T, Yasui K, Kozuka T, Towata A (2008) Protein release from yeast cells as an evaluation method of physical effects in ultrasonic field. Ultrason Sonochem 15:995-1000

110. Fernandez Rivas D, Verhaagen B, Seddon JRT, Zijlstra AG, Jiang LM, van der Sluis LWM, Versluis M, Lohse D, Gardeniers HJGE (2012) Localized removal of layers of metal, polymer, or biomaterial by cavitating microbubbles. Biomicrofluidics 6:034114

111. Fernández Rivas D (2012) Taming acoustic cavitation, PhD thesis, University of Twente, The Netherlands, Ch 7, pp 119-141

112. Ohhashi Y, Kihara M, Naiki H, Goto Y (2005) Ultrasonication-induced amyloid fibril formation of $\beta_{2}$-microglobulin. J Biol Chem 280:32843-32848

113. Carulla N, Caddy GL, Hall DR, Zurdo J, Gairí M, Feliz M, Giralt E, Robinson CV, Dobson CM (2005) Molecular recycling within amyloid fibrils. Nature 436:554558

114. Chatani E, Lee YH, Yagi H, Yoshimura Y, Naiki H, Goto Y (2009) Ultrasonication-dependent production and breakdown lead to minimum-sized amyloid fibrils. Proc Natl Acad Sci USA 106:11119-11124

115. Okumura H, Itoh SG (2014) Amyloid fibril disruption by ultrasonic cavitation: nonequilibrium molecular dynamics simulations. J Am Chem Soc 136:10549-10552 
116. Lee M, Baek I, Chang HJ, Yoon G, Na S (2014) The bond survival time variation of polymorphic amyloid fibrils in the mechanical insight. Chem Phys Lett 600:68-72

117. Tanaka K, Yamamoto K, Kadokawa JI (2014) Facile nanofibrillation of chitin derivatives by gas bubbling and ultrasonic treatments in water. Carbohydr Res 398:25-30

118. Editorial (2014) Mechanobiology in harness. Nat Mater 13:531

119. Iskratsch T, Wolfenson H, Sheetz MP (2014) Appreciating force and shape-the rise of mechanotransduction in cell biology. Nat Rev Mol Cell Biol (doi: 10.1038/nrm3903)

120. Alves-Pereira M, Castelo Branco NAA (2007) Vibroacoustic disease: biological effects of infrasound and low-frequency noise explained by mechanotransduction cellular signalling. Prog Biophys Mol Biol 93:256-279

121. Guix M, Mayorga-Martinez CC, Merkoçi A (2014) Nano/micromotors in (bio)chemical science applications. Chem Rev 114:6285-6322

122. Gao W, Wang J (2014) The environmental impact of micro/nanomachines: a review. ACS Nano 8:3170-3180

123. Hu J, Tay C, Cai Y, Du J (2005) Controlled rotation of sound-trapped small particles by an acoustic needle. Appl Phys Lett 87:094104

124. Shilton RJ, Glass NR, Chan P, Yeo LY, Friend JR (2011) Rotational microfluidic motor for on-chip microcentrifugation. Appl Phys Lett 98:254103

125. Wang W, Castro LA, Hoyos M, Mallouk TE (2012) Autonomous motion of metallic microrods propelled by ultrasound. ACS Nano 6:6122-6132

126. Takatori SC, Brady JF (2014) Swim stress, motion, and deformation of active matter: effect of an external field. Soft Matter 10:9433-9445

127. Xu T, Soto F, Gao W, Garcia-Gradilla V, Li J, Zhang X, Wang J (2014) Ultrasound-modulated bubble propulsion of chemically powered microengines. J Am Chem Soc 136:8552-8555

128. Garcia-Gradilla V, Orozco J, Sattayasamitsathit S, Soto F, Kuralay F, Pourazary A, Katzenberg A, Gao W, Shen Y, Wang J (2013) Functionalized ultrasoundpropelled magnetically guided nanomotors: toward practical biomedical applications. ACS Nano 7:9232-9240

129. Ahmed S, Wang W, Mair LO, Fraleigh RD, Li S, Castro LA, Hoyos M, Huang TJ, Mallouk TE (2013) Steering acoustically propelled nanowire motors toward cells in a biologically compatible environment using magnetic fields. Langmuir 29:1611316118

130. Wang W, Li S, Mair L, Ahmed S, Huang TJ, Mallouk TE (2014) Acoustic propulsion of nanorod motors inside living cells. Angew Chem Int Ed 53:3201-3204

131. Kagan D, Benchimol MJ, Claussen JC, Chuluun-Erdene E, Esener S, Wang J (2012) Acoustic droplet vaporization and propulsion of perfluorocarbon-loaded microbullets for targeted tissue penetration and deformation. Angew Chem Int Ed 51:7519-7522 
132. De Silva L, Yao L, Xu S (2014) Mechanically resolving noncovalent bonds using acoustic radiation force. Chem Commun 50:10786-10789

133. Valverde JM, Ebri JMP, Quintanilla MAS (2013) Acoustic streaming enhances the multicyclic $\mathrm{CO}_{2}$ capture of natural limestone at Ca-looping conditions. Env Sci Technol 47:9538-9544

134. Valverde JM, Raganati F, Quintanilla MAS, Ebri JMP, Ammendola P, Chirone R (2013) Enhancement of $\mathrm{CO}_{2}$ capture at Ca-looping conditions by high-intensity acoustic fields. Appl Energy 111:538-549

135. Gallego-Juarez JA, Riera-Franco de Sarabia E, Rodriguez-Corral G, Hoffmann TL, Galvez-Moraleda JC, Rodriguez-Maroto JJ, Gomez-Moreno FJ, Bahillo-Ruiz A, Martin-Espigares M, Acha M (1999) Application of acoustic agglomeration to reduce fine particle emissions from coal combustion plants. Env Sci Technol 33:3843-3849

136. Fernandez Rivas D, Betjes J, Verhaagen B, Bouwhuis W, Bor TC, Lohse D, Gardeniers HJGE (2013) Erosion evolution in mono-crystalline silicon surfaces caused by acoustic cavitation bubbles. J Appl Phys 113:064902

137. Virot M, Pflieger R, Skorb EV, Ravaux J, Zemb T, Möhwald H (2012) Crystalline silicon under acoustic cavitation: from mechanoluminescence to amorphization. J Phys Chem C 116:15493-15499

138. Ball P (2009) Flow-Nature's patterns. A tapestry in three parts, Oxford University Press, Ch 1, pp 1-20 